\title{
Postlearning Consolidation of Birdsong: Stabilizing Effects of Age and Anterior Forebrain Lesions
}

\author{
Michael S. Brainard and Allison J. Doupe \\ Keck Center for Integrative Neuroscience, Departments of Physiology and Psychiatry, University of California San \\ Francisco, San Francisco, California 94143-0444
}

Birdsong is a learned, sequenced motor skill. For the zebra finch, learned song normally remains unchanging beyond early adulthood. However, stable adult song will gradually deteriorate after deafening (Nordeen and Nordeen, 1992), indicating an ongoing influence of auditory feedback on learned song. This plasticity of adult song in response to deafening gradually declines with age (Lombardino and Nottebohm, 2000), suggesting that, after song learning, there continue to be changes in the brain that progressively stabilize the song motor program. A qualitatively similar stabilization of learned song can be precipitated artificially by lesions of a basal ganglia circuit in the songbird anterior forebrain (Brainard and Doupe, 2000), raising the question of whether and how these two forms of song stabilization are related. We investigated this issue by characterizing the deterioration of song that occurs after deafening in young adult birds and the degree to which that deterioration is reduced by age or by lesions of the anterior forebrain that were directed at the lateral portion of the magnocellular nucleus of the anterior neostriatum (LMAN). In most respects, LMAN lesions stabilized song to a significantly greater extent than did aging; whereas old-deafened birds eventually exhibited significant deterioration of song, lesioned-deafened birds generally did not differ from controls. The one exception was for song tempo, which was significantly stabilized by age, but not by LMAN lesions. The results indicate that LMAN lesions do not simply mimic a normal aging process, and likewise suggest that the anterior forebrain pathway continues to play a role even in the residual song plasticity that is observed after the agedependent stabilization of song.

Key words: basal ganglia; song learning; motor learning; memory consolidation; timing; language; speech; auditory feedback; hearing loss; deafness
Birdsong, like human speech, is a complex learned vocal behavior. For many songbird species, song is learned in two stages (Marler, 1970; Konishi, 1985). During a "sensory" learning period, juvenile birds memorize the sound of adult song. Then, during a "sensorimotor" learning period, they use auditory feedback to gradually match their own developing vocalizations with the stored song memory. For the zebra finch, a well studied species, sensorimotor learning is completed by $\sim 90 \mathrm{~d}$ of age, and song normally remains unchanged throughout the remainder of life. However, if auditory feedback is eliminated, song gradually deteriorates (Nordeen and Nordeen, 1992; Lombardino and Nottebohm, 2000), indicating a continuing role for hearing in the maintenance of adult song.

The susceptibility of adult song to deterioration after deafening decreases with age (Lombardino and Nottebohm, 2000). This kind of "consolidation" of learned behavior after its initial acquisition has also been observed in other cases of skilled motor learning (Dudai, 1996). A particularly striking parallel is provided by human speech; after hearing loss in adulthood, speech gradually deteriorates, sometimes eventually becoming unintelligible (Waldstein, 1990; Cowie and Douglas-Cowie, 1992). However, as

\footnotetext{
Received July 12, 2000; revised Jan. 5, 2001; accepted Jan. 5, 2001.

This work was supported by a Burroughs Wellcome Fund fellowship of the Life Sciences Research Foundation (M.S.B.) and by the John Merck Fund, the EJLB Foundation, and National Institutes of Health Grants MH55987 and NS34835 (A.J.D.). We thank N. Hessler, M. Kao, and C. Boettiger for comments on this manuscript and A. Arteseros, G. Carrillo, and A. Tam for technical assistance.

Correspondence should be addressed to Michael S. Brainard, Department of Physiology, Box 0444, 513 Parnassus Avenue, University of California San Francisco, San Francisco, CA, 94143-0444. E-mail: msb@phy.ucsf.edu.

Copyright (C) 2001 Society for Neuroscience 0270-6474/01/212501-17\$15.00/0
}

with song, the sensitivity of speech to hearing loss progressively declines over a period extending well into adulthood (Cowie and Douglas-Cowie, 1992). This declining susceptibility to disruption for both birds and humans indicates that, even after vocal production is apparently mature, it continues to be stabilized by covert changes in the brain.

The songbird anterior forebrain pathway (AFP) (Fig. 1, filled) may play an important role in regulating song plasticity. A variety of evidence suggests that the AFP is homologous to mammalian basal ganglia cortical circuits (Bottjer and Johnson, 1997; Reiner et al., 1998; Luo and Perkel, 1999). This pathway is critical for normal song learning, but unlike the song "motor pathway" (Fig. 1 , shaded), it is not required for adult song production (Nottebohm et al., 1976; Bottjer et al., 1984; Sohrabji et al., 1990; Scharff and Nottebohm, 1991; Nordeen and Nordeen, 1993; Basham et al., 1996). However, recent experiments have suggested this pathway is required for plasticity of adult song (Morrison and Nottebohm, 1993; Williams and Mehta, 1999; Brainard and Doupe, 2000); for example, AFP lesions in young adults prevent the gross deterioration of song that normally follows deafening (Brainard and Doupe, 2000).

These observations raise the question of how similar the "artificial" stabilization of song by AFP lesions is to the natural stabilization of song that occurs with aging, and whether these two phenomena are related. Here we investigate this issue by characterizing the deterioration of song that occurs after deafening in young adult birds and the degree to which that deterioration is reduced in birds that either received lesions of the AFP before deafening or that were deafened at greater ages. For most aspects of deafening-induced changes to song, lesions of the AFP 


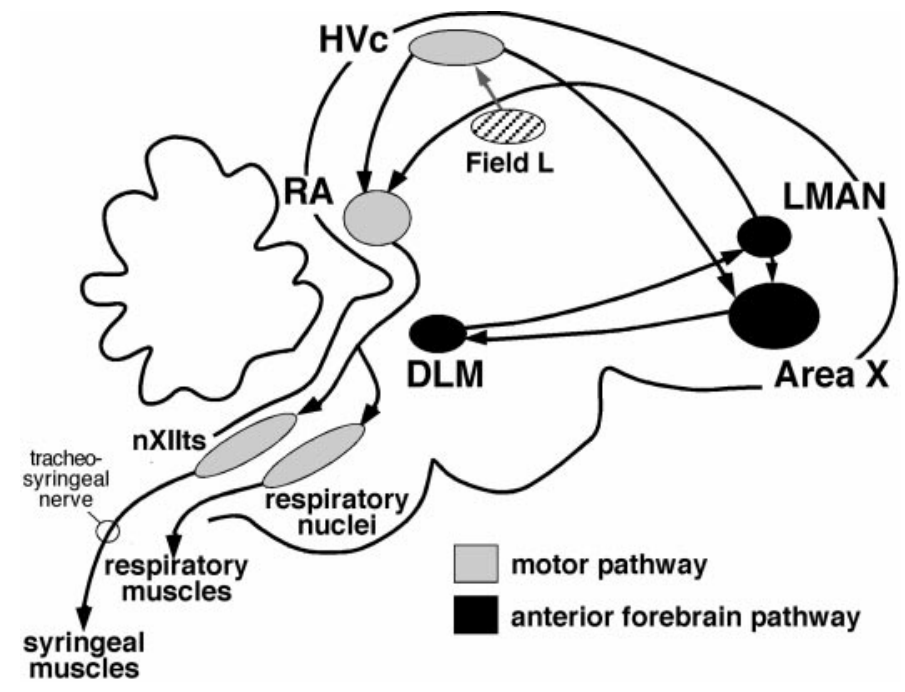

Figure 1. Schematic sagittal view of song system nuclei. The "motor pathway" (shaded) is required throughout life for song production and includes nucleus $\mathrm{HVc}$, the robust nucleus of the archistriatum (RA), and brainstem nuclei that control vocal and respiratory musculature, including the tracheosyringeal subdivision of the hypoglossal nucleus (nXIIts). The "anterior forebrain pathway" (solid) forms a basal ganglia-forebrain loop that leaves the motor pathway at the level of $\mathrm{HVc}$ and passes successively through a nucleus of the basal ganglia (Area X), the thalamus [medial subdivision of the dorsolateral thalamus $(D L M)]$, and the anterior forebrain $(L M A N)$ before returning to the motor pathway at the level of RA. Field $L$ provides auditory input to the song system via indirect connections to nucleus $\mathrm{HVc}$.

provided a greater degree of song stabilization than did aging. However, an acceleration of song tempo that followed deafening in young birds was largely prevented by aging but not by lesions of the AFP.

\section{MATERIALS AND METHODS}

\section{Animals}

The subjects for the experiments were 45 male zebra finches (Taeniopygia guttata; Table 1). Birds were raised in individual breeding cages with their parents and siblings until at least $60 \mathrm{~d}$ of age. Throughout the duration of the experiments, birds were housed with other male finches, except during song recording sessions or recovery from surgery. All birds were maintained on a 14/10 light/dark cycle throughout the experiments.

\section{Surgery}

Before all surgical procedures, birds were deprived of food for $1 \mathrm{hr}$ and then anesthetized with either Equithesin $(2 \mathrm{ml} / \mathrm{kg}$, i.m.; $0.85 \mathrm{gm}$ of chloral hydrate, $0.21 \mathrm{gm}$ of pentobarbital, $0.42 \mathrm{gm}$ of $\mathrm{MgSO}_{4}, 2.2 \mathrm{ml}$ of $100 \%$ ethanol, and $8.6 \mathrm{ml}$ of propylene glycol to a total volume of $20 \mathrm{ml}$ with water) or isoflurane (by inhalation; $2 \% \mathrm{v} / \mathrm{v}$ in $\mathrm{O}_{2}$ ). After surgery, all skin incisions were sealed with cyanoacrylate surgical glue. Birds were then transferred to individual cages equipped with heat lamps where they were monitored until full recovery before returning them to their home cages.

Birds were deafened by bilateral cochlear removal (Konishi, 1965). Complete removal of the cochlea, including the distal end of the auditory nerve, was visually confirmed using a dissecting microscope. After cochlear removal, some birds showed signs of vestibular disturbance that usually resolved in the first few days after surgery. Extra care was taken to ensure that such birds had easy access to seed and maintained full crops. Birds did not exhibit difficulty in perching, feeding, or interacting with other birds after returning to their home cages.

Electrolytic lesions were stereotaxically targeted at the lateral portion of the magnocellular nucleus of the anterior neostriatum (LMAN), with up to five penetrations per side and two current injections per penetration $(100 \mu \mathrm{A}$ for $60 \mathrm{sec})$. For all but one of the nominally lesioned birds, the percentage of LMAN that was removed bilaterally ranged from 70 to
$100 \%$. The remaining bird had only a $37 \%$ lesion, but it included the region where robust nucleus of the archistriatum (RA) projecting axons leave LMAN, and almost all calcitonin gene-related peptide (CGRP) labeling (see below) in RA was eliminated, indicating that the afferent projection from LMAN to RA had been interrupted. "Sham" lesions were entirely anterior and dorsal to LMAN.

All procedures were performed in accordance with established animal care protocols as approved by the University of California San Francisco Committee on Animal Research.

\section{Anatomy}

After the final song recording session, birds were deeply anesthetized with Metofane (by inhalation; Pitman-Moore, Mundelein, IL) and transcardially perfused with $0.9 \%$ saline, followed by $3.7 \%$ formalin in 0.025 M phosphate buffer. Brains were post-fixed for $4 \mathrm{hr}$, then sunk in sucrose overnight and cut coronally in $40 \mu \mathrm{m}$ sections with a freezing microtome. Alternate sections were stained with cresyl violet acetate or reacted with an antibody to CGRP (Bottjer et al., 1997).

\section{Song analysis}

Sound recording. All song was recorded from birds that were isolated in sound attenuating chambers and therefore was "undirected." A computerized, song-activated recording system was used to detect and digitize song for later off-line analysis using software written in the Matlab programming language (Mathworks). For each bird and time point, $\geq 10$ bouts of song were recorded for analysis.

Song structure. "Bouts" of song were defined as periods of singing surrounded by silent intervals at least $2 \mathrm{sec}$ in duration. "Syllables" were defined as song elements that were separated from each other by silent intervals exceeding $5 \mathrm{msec}$ in duration. Typical bouts of song started with a variable number of short introductory syllables followed by one or more "motifs", consisting of stereotyped sequences of more complex syllables. For some birds, there were one or two variants on a basic motif; these typically differed in the presence or absence of optional syllables, usually occurring at the end of a conserved "core" sequence. We defined a single characteristic motif for each bird as the motif variant that occurred most frequently across all recorded songs. We defined the "repertoire" of syllables for a bird as all structurally distinct syllables (including introductory syllables) that were present in at least $50 \%$ of a bird's song bouts.

Syllable similarity. To characterize changes to the structure of individual syllables, we used a subjective scoring procedure similar to that used in previous studies of birdsong (Nordeen and Nordeen, 1992; Morrison and Nottebohm, 1993; Brainard and Doupe, 2000; Lombardino and Nottebohm, 2000). Three observers that were blind to the experimental manipulation of each bird scored the similarity between spectrographic representations of syllables from songs recorded before any manipulation and those from later songs. The observers were all highly familiar with spectrographic representations of zebra finch song. One of the observers had some previous experience with spectrograms of the birds in the study and was aware of the design of the experiment, whereas the other two observers had little or no previous experience with the spectrograms or the design of the experiment. During the scoring procedure, song identity was encoded so that observers were unaware of the treatment of the birds from which the songs were recorded. To minimize any order effects, scoring was performed in blocks in which pairs of songs were drawn from each treatment group and randomly interleaved. For each baseline syllable that was initially present in the repertoire of a bird (5-12 distinct syllables per bird), observers identified the most similar syllable present in songs recorded at later dates. Observers additionally judged the degree of similarity between each baseline syllable and its best match on a scale of 0 (no similarity) to 3 (identical). Scores were averaged across the three observers. The mean correlation coefficient for pairwise comparisons of observer's scores was 0.91 , indicating a similar degree of interobserver reliability to a previous study, which reported a correlation coefficient between two observer's scores of 0.95 (Lombardino and Nottebohm, 2000). The significance of each of the differences between groups reported in Results was not affected by the exclusion of scores from any one observer. Because observers based their judgments on spectrographic representations of syllables that were presented individually, this measure emphasized changes in the structure of syllables rather than changes in the sequences in which syllables were sung or the overall temporal pattern of song. In this respect our measure of syllable similarity differed slightly from the "song similarity index" used by Lombardino and Nottebohm (2000), in which observers were presented with intact segments 


\begin{tabular}{|c|c|c|c|c|c|}
\hline & $\begin{array}{l}\text { Young control } \\
n=9(4,9)^{a}\end{array}$ & $\begin{array}{l}\text { Old control } \\
n=8(5,5)^{a}\end{array}$ & $\begin{array}{l}\text { Young deaf } \\
n=11(10,10)^{a}\end{array}$ & $\begin{array}{l}\text { Old deaf } \\
n=8(8,6)^{a}\end{array}$ & $\begin{array}{l}\text { Lesioned and deaf } \\
n=6(6,6)^{a, c}\end{array}$ \\
\hline \multicolumn{6}{|l|}{ Syllable similarity } \\
\hline 1 month & $2.76(0.05)^{b}$ & $2.9(0.02)$ & $1.71(0.21)^{*}, * *$ & $2.63(0.08)$ & $2.49(0.16)^{* * *}$ \\
\hline$>180 \mathrm{~d}$ & $2.56(0.12)$ & $2.85(0.03)$ & $1.22(0.21)^{*}, * *$ & $1.84(0.19)^{*}$ & $2.33(0.18)^{* * *}$ \\
\hline \multicolumn{6}{|l|}{ Temporal similarity } \\
\hline 1 month & $0.71(0.04)$ & $0.80(0.06)$ & $0.33(0.1)^{*}, * *$ & $0.58(0.11)$ & $0.8(0.02)^{* * *}$ \\
\hline$>180 \mathrm{~d}$ & $0.70(0.05)$ & $0.79(0.027)$ & $0.20(0.1)^{*}$ & $0.26(0.12)^{*}$ & $0.75(0.03)^{* * * * * *}$ \\
\hline Modulation $>180 \mathrm{~d}$ & $-0.51(0.17)^{* * * *}$ & $0.04(0.24)$ & $1.78(0.63)^{*}$ & $0.93(0.29)$ & $0.27(0.37)^{* * *}$ \\
\hline Noisiness $>180 \mathrm{~d}$ & $-0.20(0.09)$ & $0.07(0.09)$ & $0.67(0.13)^{*}$ & $0.74(0.21)^{*}$ & $0.14(0.06)^{* * * * *}$ \\
\hline Waveriness $>180 \mathrm{~d}$ & $-0.14(0.08)$ & $0.05(0.11)$ & $0.82(0.17)^{*}$ & $0.82(0.17)^{*}$ & $0.16(0.11)^{* * * * * *}$ \\
\hline$\%$ abnormal $>180 \mathrm{~d}$ & $-1.4(1.67)$ & $3.2(1.79)$ & $39.9(7.9)^{*}$ & $30.1(8.0)^{*}$ & $1.95(0.94)^{* * * * * *}$ \\
\hline Fund. freq. $>180 \mathrm{~d}$ & $102(0.7)$ & $99(1.3)$ & $101(1.8)$ & $100(2.2)$ & $100(1.2)$ \\
\hline All syllable durations $>180 \mathrm{~d}$ & $93.8(2.9)$ & $104.8(1.4)$ & $69.7(8.3)^{*}$ & $75.9(4.6)^{*}$ & $78.5(2.0)$ \\
\hline All interval durations $>180 \mathrm{~d}$ & $86.3(5.4)^{* * * *}$ & $95.4(10.6)$ & $150.3(10.8)^{*}$ & $147.5(24.3)^{*}$ & $105.4(13.4)^{* * *}$ \\
\hline \multicolumn{6}{|l|}{ Matched total durs. } \\
\hline 1 month & $98.5(3.2)$ & $100.3(0.8)$ & $93.9(2.4)$ & $97.6(1.2)$ & $91.2(2.0)^{*}, * *$ \\
\hline$>180 \mathrm{~d}$ & $93.2(5.7)^{* * * *}$ & $98.4(0.9)$ & $82.3(4.0)^{*}, * *$ & $96.0(3.4)$ & $80.1(2.2)^{*}, * *$ \\
\hline \multicolumn{6}{|l|}{ Matched syll. durs. } \\
\hline 1 month & $98.9(4.8)$ & $101.1(1.3)$ & $90.9(2.1)^{*}$ & $96.7(1.7)$ & $92.2(3.6)$ \\
\hline$>180 \mathrm{~d}$ & $94.5(3.6)^{* * * *}$ & $100.01(0.2)$ & $81.53(2.9)^{*}, * *$ & $95.7(4.1)$ & $80.9(2.8)^{*}, * *$ \\
\hline \multicolumn{6}{|l|}{ Matched int. durs. } \\
\hline 1 month & $98.2(7.1)$ & 96.9 (1.9) & $117.8(8.1)$ & $109.9(5.1)$ & $90.0(7.4)^{* * * * * *}$ \\
\hline$>180 \mathrm{~d}$ & $89.6(14.5)$ & $92.9(3.6)$ & $108.6(5.6)^{*}$ & $105.3(8.5)$ & $85.1(7.5)^{* * * * * *}$ \\
\hline
\end{tabular}

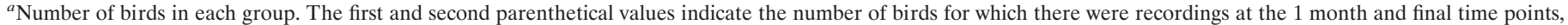

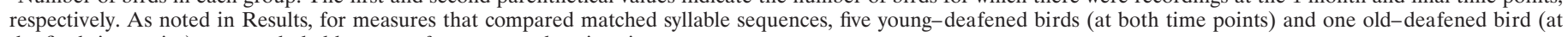
the final time point) were excluded because of gross song deterioration.

${ }^{b}$ Values indicate means and SEs for each measure.

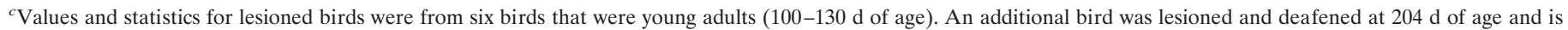
included in figures with a separate symbol.

*Indicates significant difference from age-matched controls.

**Indicates significant difference between young-deafened and old-deafened birds or between lesioned birds and old-deafened birds.

***Indicates significant difference between lesioned birds and young deafened birds.

**** Indicates significant change between pre and post values for control birds.

of song from recordings before and after deafening and were instructed to explicitly consider sequence information in comparing syllables.

For many of the deafened birds, the variable nature of syllables present in songs after deafening made it impossible to define a fixed repertoire of syllables. Therefore, for these birds not all syllables from deteriorated (postdeafening) songs were presented as possible matches to syllables from the bird's initial repertoire. However, care was taken to include in the panel of potential matches a representative assortment of syllables as well as any syllables that bore resemblance to syllables from the predeafening repertoire, even if they occurred with very low frequency.

We established an approximate range for the syllable similarity measure by having observers score syllable similarity for pairs of songs recorded from control birds as well as for pairs of unrelated songs. A maximum expected syllable similarity score was established by comparing songs recorded on the same date from the same bird (i.e., no elapsed time between recordings); the mean syllable similarity score for this nominally unchanged group was 2.85 ( $\mathrm{SE}=0.09 ; n=6$ birds, three control and three deafened). To establish a minimum value for syllable similarity, we had observers score the similarity of syllables from pairs of unrelated songs. Although some individual syllables from the "pre" repertoire (recorded from one bird) were scored as having no similarity to syllables present in the "post" songs (recorded from an unrelated bird), this was not usually the case. The mean syllable similarity score for unrelated pairs of songs was $0.96(\mathrm{SE}=0.08 ; n=20,10$ pairs of control songs and 10 pairs of songs from deafened birds).

Changes to syllable spectral structure. A qualitative examination of songs in this and previous studies (Nordeen and Nordeen, 1992; Wang et al., 1999) suggested that the spectral structure of syllables deteriorates after deafening. We used both subjective and objective measures to assess this impression.

When it was possible to follow individual syllables of a bird's repertoire over time, we used an automated fitting procedure to measure changes to the fundamental frequency of selected syllables and to the noisiness of those syllables as reflected by the depth of harmonic modulation. Log power spectra were calculated for $16 \mathrm{msec}$ samples of the syllable sound waveform. A modified discrete cosine-transform of the spectrum was used to identify the fundamental frequency: this procedure essentially calculated, for each frequency, $F$, in the range $300-1200 \mathrm{~Hz}$ (with $2 \mathrm{~Hz}$ increments), the average difference in height between spectral peaks that were harmonically related to $F$ (at frequencies of $n^{*} F$ ) and intervening troughs [at frequencies of $(n+0.5)^{*} F$ ]. Because energy in zebra finch song is concentrated at frequencies $<8 \mathrm{kHz}$, this difference was calculated only for peak locations $<7 \mathrm{kHz}$ ( $n$ was constrained to be $<7000 / F$ ). The fundamental frequency was defined as the value of $F$ that maximized this peak-trough difference, and the depth of modulation was defined as the value of this difference at the fundamental frequency. This depth of modulation measure is larger to the extent that there is a well defined harmonic structure to the spectrum, and smaller to the extent that the spectrum is "flatter", with a more uniform distribution of energy across frequency.

We applied this procedure to individual syllables that had clear harmonic structure with a well defined fundamental frequency and that remained recognizable after deafening, to quantify any systematic changes to these parameters. Measurements were made only on constant frequency components of syllables so that any variation (across exemplars of the syllable) in the location within the syllable that was selected for analysis would have minimal influence on measured fundamental frequency. For each syllable the fundamental frequency and depth of modulation were represented by the median value of measurements made across five exemplars. For each bird, the changes to these parameters were represented by the median of changes for all syllables that were measured (one to five syllables per bird). 
A 103 days old

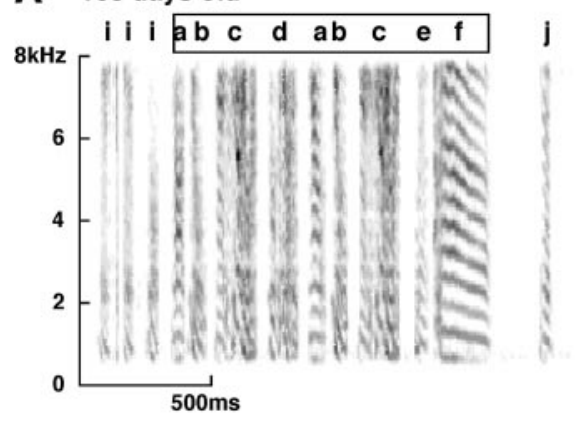

\section{B 540 days old}

Figure 2. Example illustrating the stable song of a normal adult zebra finch (zfa12). $A$ shows a spectrographic representation of song recorded from a normal zebra finch at $103 \mathrm{~d}$ of age. $B$ shows an example of the same bird's song recorded at $540 \mathrm{~d}$ of age. Individual syllables of the bird's repertoire are labeled above each song; boxes enclose the stereotyped sequence of syllables, or motif, that characterized the bird's song. Both the repertoire of syllables and the order in which they were sung remained essentially unchanged, although the rate of song delivery increased.

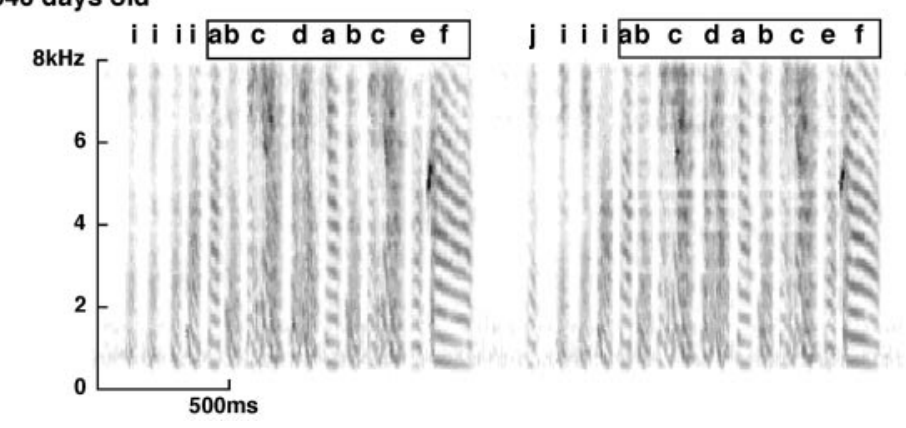

To assess changes to the spectral quality of syllables, independently of whether individual syllables remained recognizable over time, we also used a subjective scoring procedure. Observers who were familiar with zebra finch song but blind to the treatment of individual birds scored the overall "noisiness" of the harmonic structure and the "waveriness" of the fundamental frequency (see below) of 50-100 syllables randomly drawn from each recording session. Observers additionally judged whether or not each syllable appeared to be from a normal zebra finch song. Judgments were based on spectrographic representations of syllables that were presented in isolation. The scoring procedure was performed in blocks in which syllables from birds that received different treatments were randomly interleaved to ensure that observers remained blind to the treatment group from which individual syllables were drawn.

For each syllable, observers scored noisiness on a $0-3$ scale. Noisiness scores were based on the degree to which harmonically related frequencies were well defined. Syllables that received noise scores of 0 had well defined fundamental frequency for the entire duration of the syllable and had spectra that were deeply modulated so that power was concentrated at harmonically related frequencies (e.g., syllables "a" and "f" in Fig. $2 a$ and syllables "a", "b", "e", and "g" in Fig. 5a). At the opposite extreme, syllables that received scores of 3 had essentially no identifiable fundamental frequency over most of their duration and had spectra with a fairly uniform distribution of power across frequency (e.g., the final syllables in Fig. $3 d$ and syllable "a" in Fig. $4 d$ ).

Observers also scored syllable waveriness on a $0-3$ scale. This measure was intended to document what appeared to be abnormally rapid fluctuations in frequency of some syllables sung by birds after deafening. Syllables that received a waveriness score of 0 had component stacks or sweeps for which the fundamental frequency changed smoothly over time (e.g., syllables "a" and "b" in Fig. 5a). In contrast, those syllables that received a waveriness score of 3 had abnormally rapid fluctuations in fundamental frequency over the course of component stacks or sweeps (e.g., syllable "d" in Fig. 4c). Judgments of waveriness were based only on those portions of syllables in which the fundamental frequency was best defined; hence, for very noisy syllables in which the fundamental frequency was not apparent, no score was assigned.

Changes to the temporal pattern of song. To assess temporal stability, songs were represented by the timing of syllables and intervals, whereas the spectral structure of individual syllables was ignored. For each bird the timing pattern representing the most common motif before any experimental manipulation was identified. We then searched song bouts from later recording sessions, using a cross-correlation-based technique, for the pattern of syllables that provided the closest temporal match to the initial motif: at each possible alignment (in increments of $5 \mathrm{msec}$ ) between motif and song bout, the degree of temporal match was quantified as the average of the percentage overlap of syllables and the percentage overlap of intervals. Interval overlap and syllable overlap were calculated separately and then averaged to increase the sensitivity of the measure to the pattern of song: intervals constitute only a small fraction of the overall song motif (and hence would influence a simple cross-correlation of syllables and intervals combined only minimally), but their location is a critical component of the temporal pattern. For each bird, the maximal overlap was calculated for 10 song bouts and then averaged to provide a measure ("xcorr") of how well the temporal pattern of song was conserved after experimental manipulation. Because the delivery of some songs speeded up "proportionately" over time (i.e., with no apparent change in the relative durations of syllables and intervals), we allowed for proportional changes in the temporal pattern of the song (ranging from 75 to $110 \%$ of initial song duration) in searching for the maximal overlap. To control partially for the varying complexity of the motifs sung by different birds, the same method was used to calculate the maximal overlaps between each motif and one song bout from each of 20 different unrelated birds; these 20 different values of maximum overlap were averaged to give a measure ("rand") of the expected similarity between each motif and random, unrelated songs. The temporal stability of song was then expressed as a normalized value ranging from 0 (indicating no more preservation of temporal pattern than random) to 1 (indicating perfect preservation of temporal pattern):

$$
\text { temporal similarity }=(\text { xcorr }- \text { rand }) /(1-\text { rand }) .
$$

The baseline motif for one bird from the deafened group and one bird from the lesioned-deafened group had a very simple temporal pattern (three syllables) and consequently showed a high degree of temporal similarity to all songs (including those from unrelated birds). These two birds were therefore excluded from further analysis of changes in temporal pattern.

Syllable and interval durations and song tempo. At least two methods have previously been used to characterize changes to syllable and interval durations. Several studies have first defined the repertoire of syllables for each set of songs, and then defined the mean syllable duration as the average of a single instance of each (unique) element of the repertoire (Price, 1979; Scharff and Nottebohm, 1991; Nordeen and Nordeen, 1992). Changes in this measure after a manipulation (such as deafening) presumably may reflect any changes in the durations of individual elements that are conserved (i.e., present in the repertoire both before and after the manipulation), as well as changes in which syllables are assigned to the repertoire. For example, the dropping of a relatively long duration 
A pre-deafening (105days old)

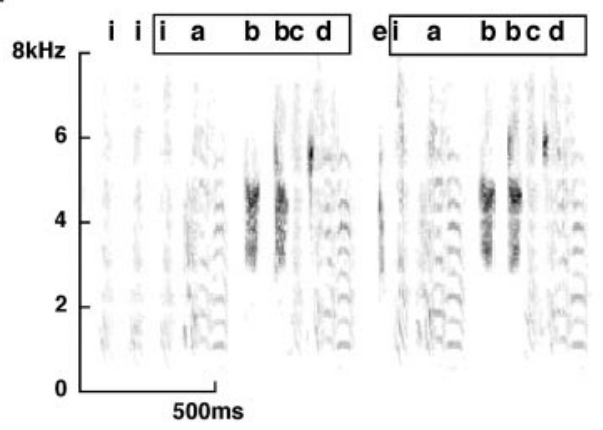

B 3 days post-deafening
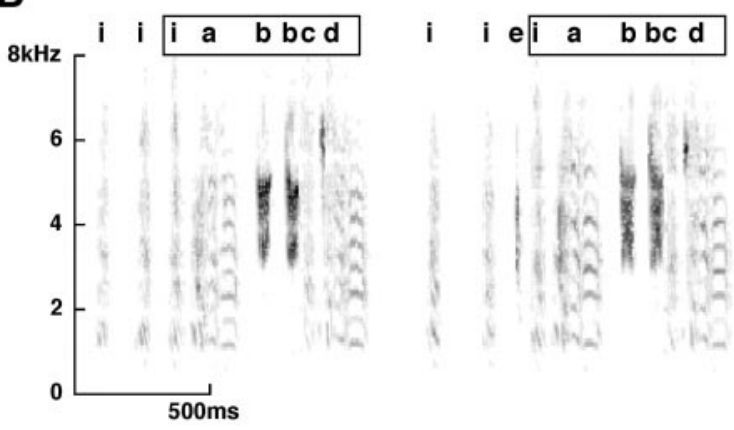

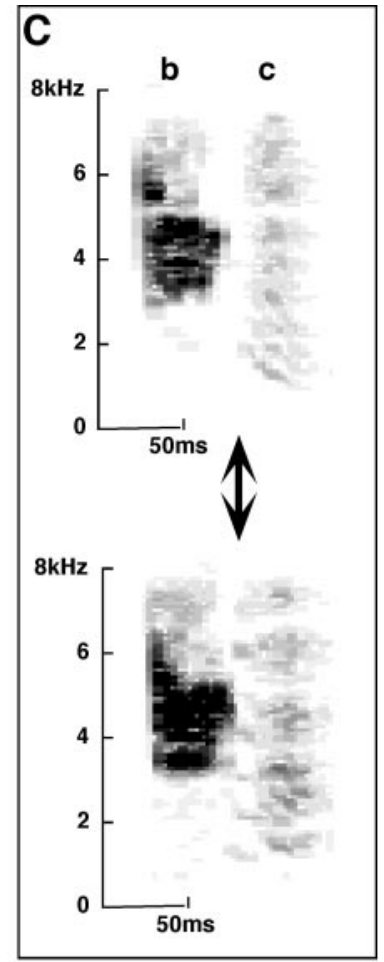

D 26 days post-deafening
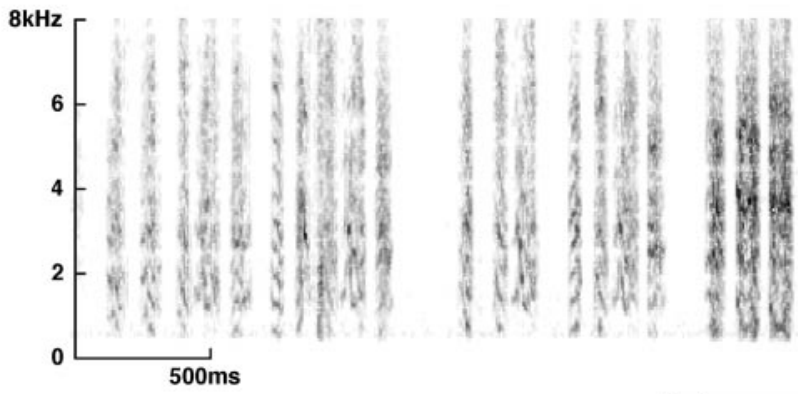

E

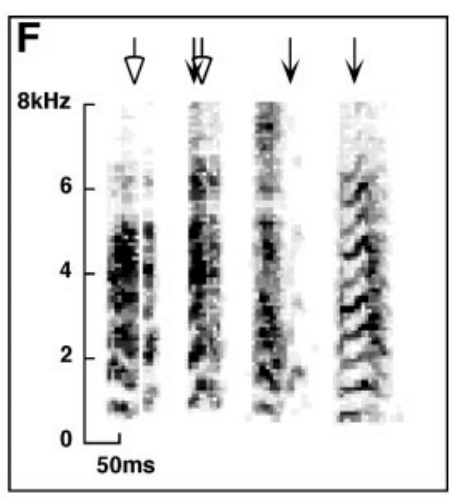

Figure 3. Example illustrating one of the larger changes to song observed after deafening (zfa4), in which the original song degraded to an unrecognizable state. $A$, Song at $105 \mathrm{~d}$ of age, immediately before deafening. $B$, Song from $3 \mathrm{~d}$ after deafening. $C$, Expanded time axis showing an example of syllables "b" and "c" recorded before (top) and $3 \mathrm{~d}$ after (bottom) deafening. After deafening the previously silent interval between the syllables was often "voiced" (arrow). $D$, Song from $26 \mathrm{~d}$ after deafening. None of the original repertoire of syllables remained recognizable at this date. $E$, Song from $475 \mathrm{~d}$ after deafening. Syllables had further degraded and now included features not normally observed in zebra finch songs from our colony. $F$, Expanded time axis showing examples of some abnormal syllables from songs recorded $475 \mathrm{~d}$ after deafening. These included upwardly sweeping frequency components (solid arrows) and brief silent intervals intercalated within short duration syllables (open arrows). syllable from the repertoire would contribute to a shortening of mean syllable duration. A second method has been to determine the mean duration of syllables (and intervals) by including every instance of all syllables (and intervals) sung by a bird in a given set of songs, without regard to the identity of syllables (Wang et al., 1999). This measure will also be influenced by any changes in the durations of conserved song elements as well as by changes in the relative probability with which elements of differing durations appear in the song. For example, increased repetition of short-duration syllables would contribute to a shortening of mean syllable duration. For many birds in the current study, we could not confidently define a fixed repertoire of syllables in the songs recorded after deafening. We therefore used the latter measure in characterizing changes to mean syllable and interval durations. So that our data would be directly comparable with the previous study that used this measure, we included only intervals that were $<500 \mathrm{msec}$. In Results, we refer to the song elements to which this measure was applied as "unmatched syllables" and "unmatched intervals".

In cases in which identified sequences of syllables were recognizably retained, we also measured changes in "song tempo", or the rate at which those matched sequences were delivered (Sossinka and Bohner, 1980; Williams and Mehta, 1999). In Results, we separately report changes to the durations of the "matched syllables" and "matched intervals" that constitute these retained sequences. For these analyses we only considered sequences in which three or more contiguous syllables were retained.

Statistics. For each measurement and time point we used an ANOVA 
A pre-deafening (103 days old)

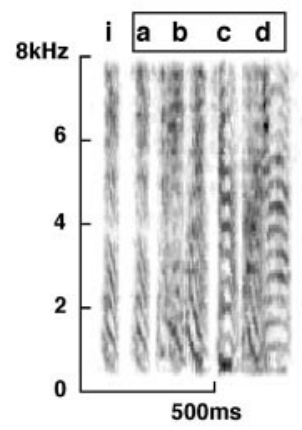

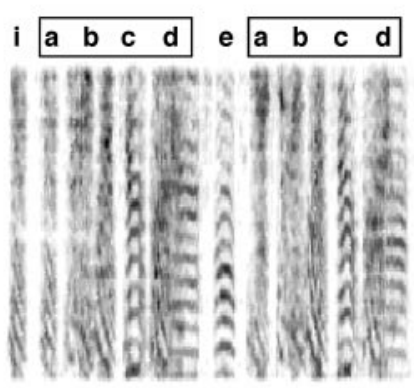

C 41 days post-deafening
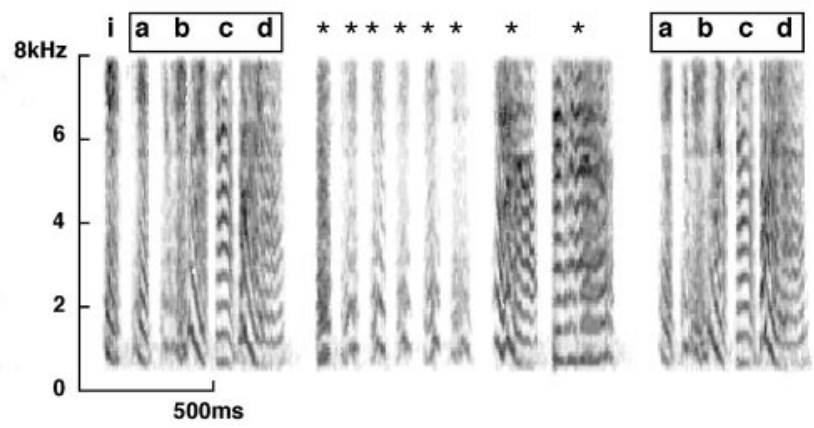

B 7 days post-deafening

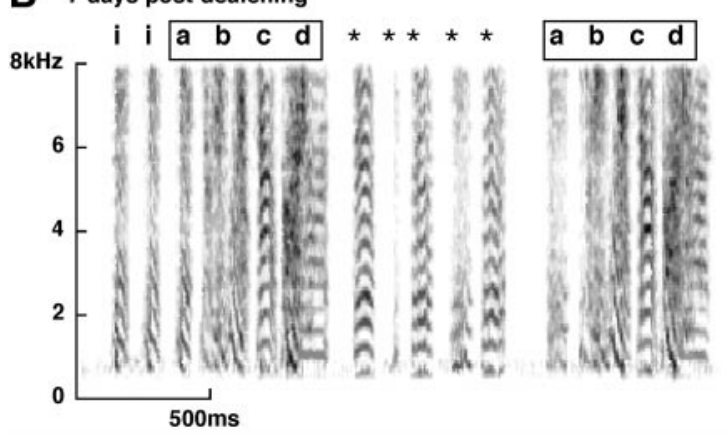

D 203 days post-deafening

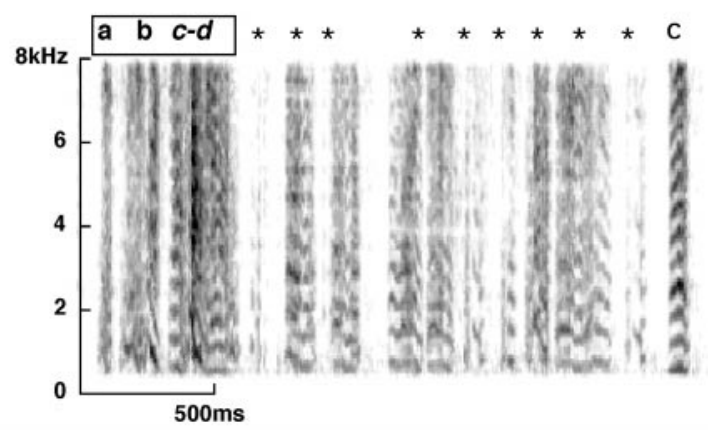

Figure 4. Example illustrating one of the smaller changes to song observed after deafening (zfa7), in which all original syllables remained recognizable but were spectrally degraded, and in which abnormal vocalizations were introduced into the song. $A$, Song at $103 \mathrm{~d}$ of age, immediately before deafening. $B$, Song from $7 \mathrm{~d}$ after deafening. The original syllables were preserved, but variable, low-amplitude vocalizations were also introduced into the song $(*)$. $C$, Song from $41 \mathrm{~d}$ after deafening. In addition to the presence of abnormal vocalizations $(*)$, the spectral structure of original syllables had begun to degrade. $D$, Song from $203 \mathrm{~d}$ after deafening. The spectral structure of original syllables had further degraded, and syllables "c" and "d" had become merged.

to test for significant effects of experimental treatment (Table 1). A post hoc test (Fischer's protected least significant difference) was used to determine the significance of differences between pairs of experimental treatments. Table 1 summarizes comparisons and indicates those for which significance was achieved at $p<0.05$.

\section{RESULTS}

The deterioration of song after deafening has been previously described, particularly with respect to syllable changes (Nordeen and Nordeen, 1992; Wang et al., 1999; Lombardino and Nottebohm, 2000). Here we provide an additional description of changes in song after deafening, focusing on both syllable structure and the global temporal organization of song. This characterization was a prerequisite to a detailed comparison of the stabilizing effects of age and AFP lesions. We observed deafening-induced changes that included deterioration of syllables, loss of syllables, addition of abnormal material, and alterations to the temporal pattern of song, including changes to syllable sequencing and to tempo. To qualitatively illustrate the range of changes that we observed, we first present examples of songs recorded before and at varying intervals after deafening. In the remainder of the results we examine more systematically and quantitatively these changes and how they were influenced by the age at which birds were deafened and by lesions of the anterior forebrain nucleus LMAN before deafening.

\section{Control birds}

Manipulations were performed on adult male zebra finches that were $\sim 100 \mathrm{~d}$ of age or older. Previous studies of the development of zebra finch song have reported that by this age individual birds have learned a fairly stable and stereotyped song ("crystallized song") that they will maintain with little modification throughout life (Immelmann, 1969; Arnold, 1975; Scharff and Nottebohm, 1991; Bottjer and Hewer, 1992; Williams and Mehta, 1999; Lombardino and Nottebohm, 2000). Our recordings from normal (control) zebra finches were generally consistent with this view. By $100 \mathrm{~d}$ of age, each bird had developed a fixed repertoire of 5-12 distinct syllables that were typically sung in a characteristic sequence with little variation from one song rendition to the next. Figure $2 a$ illustrates a segment of song sung by a control zebra finch at $103 \mathrm{~d}$ of age. This bird had a repertoire of eight distinct syllables that together accounted for essentially all of the bird's vocalizations during song. After a variable number of introductory syllables ("i" and "j") the bird typically sang a fixed, characteristic sequence or motif ("abcdabcef"), although in some renditions there were variations such as the truncation of the sequence before the two terminal syllables (data not shown).

For control birds, the initial syllable repertoire and characteristic sequencing of syllables generally changed little over time. Figure $2 b$ shows a segment of song recorded at $540 \mathrm{~d}$ of age from the same bird. Although $>1$ year had elapsed from the initial recording, each of the syllables that was initially present was still clearly identifiable and there were at most only subtle changes in the structure of individual syllables. Likewise, the typical sequence in which the syllables were sung remained unchanged.

Although the syllable structure and syllable sequencing of control songs recorded at $\sim 100 \mathrm{~d}$ of age generally remained un- 
A pre-deafening (395 days old)

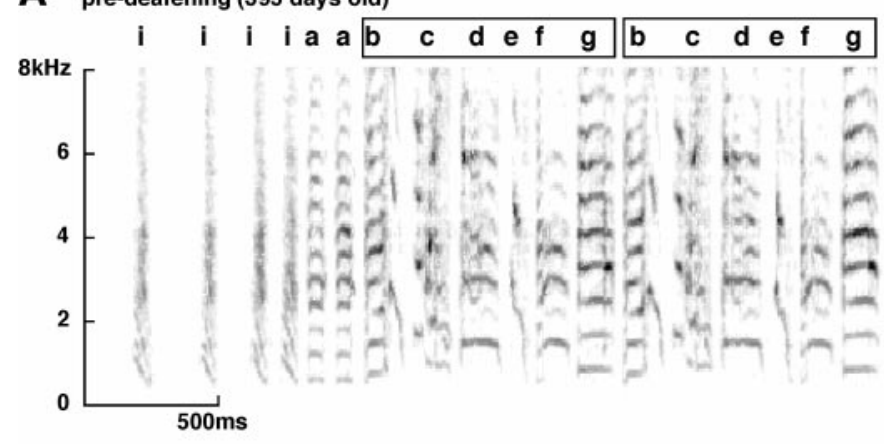

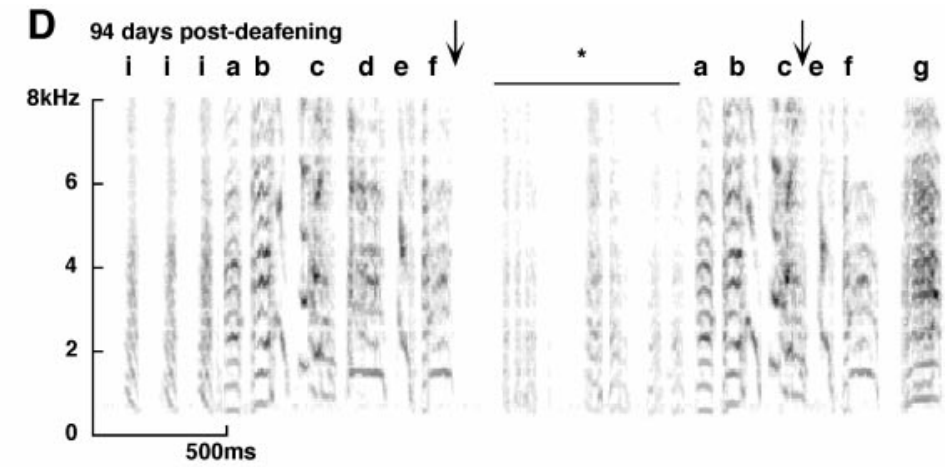

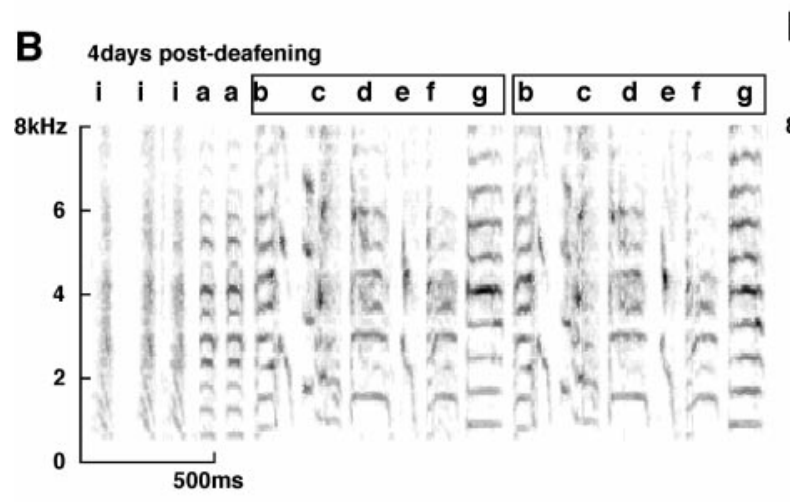

E 256 days post-deafening
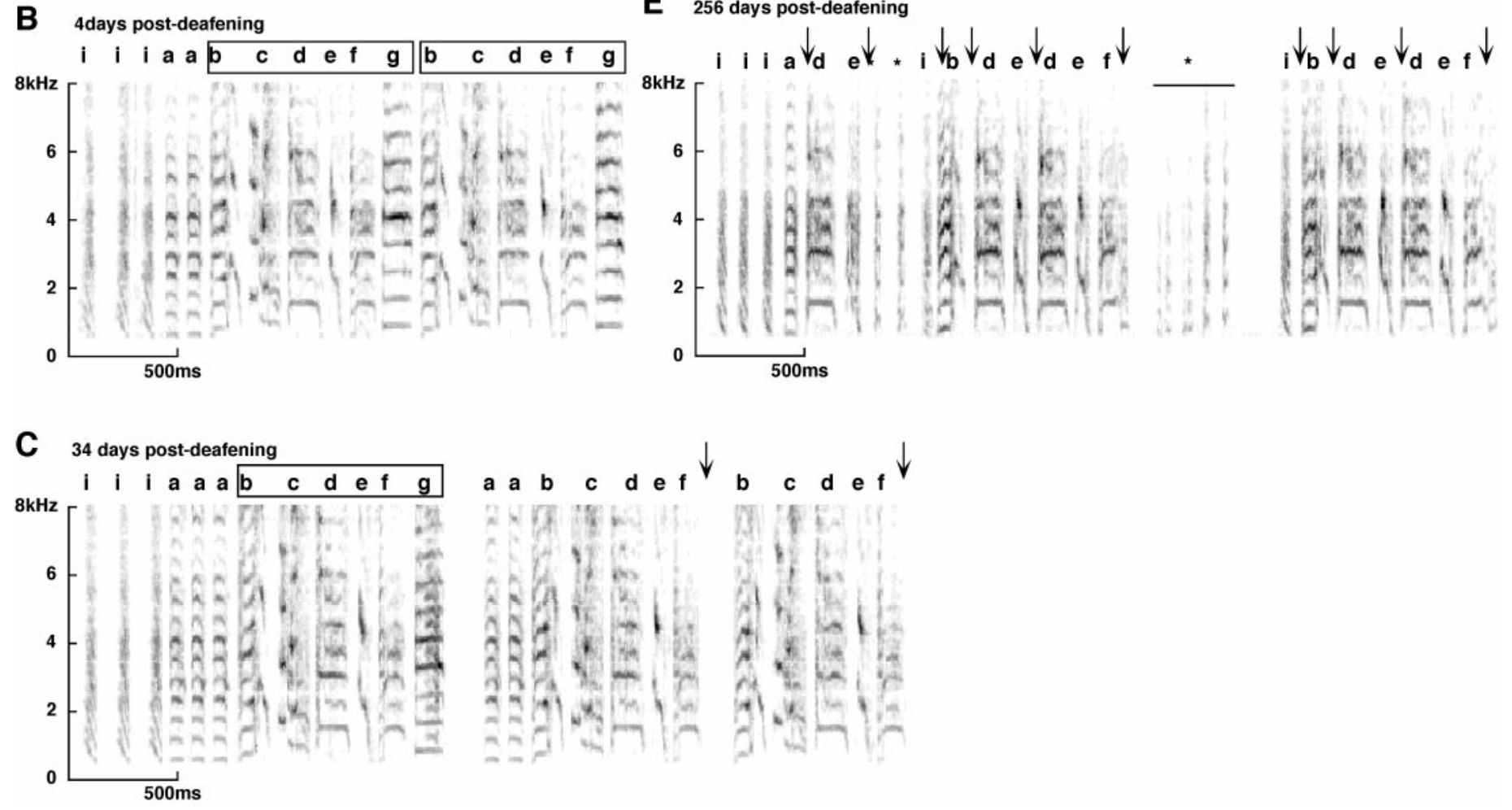

Figure 5. Another example illustrating one of the smaller changes to song observed after deafening (zfm1), in which some original syllables remained recognizable but exhibited changes both to spectral structure and to sequencing. $A$, Songs recorded at $395 \mathrm{~d}$ of age, before deafening. $B$, Songs from $4 \mathrm{~d}$ after deafening. $C$, Songs from $34 \mathrm{~d}$ after deafening. Syllable "g" was frequently dropped from the song (arrows) and, when sung, was spectrally degraded. $D$, Songs from $94 \mathrm{~d}$ after deafening. In addition to further degradation and dropping of syllable "g", songs at this date also included other abnormalities of sequence (arrows) and introduction of abnormal syllables (*). E, Songs from $256 \mathrm{~d}$ after deafening. Songs exhibited a further degradation of spectral structure, more new transitions between recognizable syllables (arrows), and continued presence of abnormal syllables $(*)$.

changed at later ages, there were for some birds more subtle changes in song. For example, over time the frequency structure of some individual syllables appeared to become less noisy and variable, suggesting that control of the detailed structure of syllables continued to improve with age. Likewise, in some birds there were small changes with age in the relative probability with which different syllable sequences were sung. Finally, in many birds, there was a speeding up of song production with age so that the same syllables were delivered more rapidly. This is apparent for the songs in Figure 2, in which the total duration of the sequence "abcdabc" decreased from $867 \mathrm{msec}(\mathrm{SD}=14 \mathrm{msec})$ at $103 \mathrm{~d}$ of age to $736 \mathrm{msec}(\mathrm{SD}=5 \mathrm{msec})$ at $540 \mathrm{~d}$ of age. Apart from these sorts of subtle changes, songs of control birds were largely unchanging beyond $\sim 100 \mathrm{~d}$ of age.

\section{Young deafened birds}

In contrast, the songs of adult birds that were deafened could change dramatically. As in humans, however, the degree of change in vocal output after deafening was quite variable across individuals. Figure 3 illustrates one of the more extreme examples of deafening-induced changes in song, observed in a bird that was deafened at $105 \mathrm{~d}$ of age. Immediately before deafening, the bird had a well defined repertoire of six different syllables (including introductory syllables) that were generally sung in a stereotyped sequence ("iabbcd"; Fig. 3a). Three days after deafening, the bird's song was largely unchanged (Fig. $3 b$ ); each of the syllables that was present initially was retained recognizably with little or no modification. However, the syllable "e" which, before deafening, was usually interposed between occurrences of the sequence 
"iabbcd", was rarely sung after deafening. Furthermore, the interval between syllables " $b$ " and "c", which had originally been silent, was frequently "voiced" after deafening (Fig. 3c), suggesting a possible disruption in the coordination of vocal and respiratory musculature. The lack of dramatic changes in the song of this bird immediately after deafening was typical of birds in this study.

By $26 \mathrm{~d}$ after deafening, the original repertoire of syllables sung by this bird was no longer apparent (Fig. 3d). Conspicuously absent were the distinctive high-frequency syllables " $b$ " and "e" as well as the complex syllable "d". Most of the syllables present at this point did not have clear antecedents in the predeafening song, and many of the syllables appeared to be noisy, with poorly defined harmonic structure. Because we could not establish correspondences between the syllables that were sung before and after deafening, it was not possible to assess the degree to which there had been further changes in the sequencing of syllables. The detailed temporal pattern of the song, however, appeared to differ qualitatively from that present before deafening, suggesting that the changes in song reflected more than just an alteration of the spectral structure of individual syllables.

By the final recording from this bird, at more than a year after deafening (Fig. 3e), the syllables had deteriorated further, and many exhibited features that were not found in normal syllables recorded from birds in our colony (Fig. 3f). These abnormal features included frequency components that swept upwards (sol$i d$ arrows) and brief silent intervals ( $<5 \mathrm{msec}$ duration) that split short duration syllables into two separate components (open arrows). The overall temporal pattern of the song had also changed further to a series of relatively uniform, short syllables.

The rapid and dramatic changes in song that were seen for this bird were shared by several of the other birds that were deafened at $\sim 100 \mathrm{~d}$ of age. For these birds, the syllables originally present in the song were rapidly obscured by deafening-induced changes, so that they were no longer recognizable in songs recorded as early as a few weeks after deafening.

At the opposite extreme, there were birds that exhibited comparatively minor changes in song and retained recognizable versions of their original syllables even months after deafening. Figure 4 illustrates changes in song for one such bird. This bird was deafened at $105 \mathrm{~d}$ of age. Before deafening, the bird regularly sang the four syllable sequence "abcd". At 7 and $41 \mathrm{~d}$ after deafening, the bird still sang a relatively unchanged and easily recognizable version of this sequence (Fig. 4b,c). Indeed, even at 6 months after deafening, the bird continued to produce a modified but recognizable version of the sequence, in which the most notable change was that the terminal two syllables ("c" and " $d$ ") were merged (Fig. 4d).

Despite the preservation of this characteristic syllable sequence, there were nevertheless a variety of appreciable changes to the bird's song that followed deafening. These included the introduction of syllables to song that had not been present in the initial repertoire (*). These syllables often had an abnormal structure and were of lower amplitude than the more normal and recognizable syllables. These introduced syllables were often separated by intervals of longer duration than typically occur within the context of a motif. Unlike the syllables of control songs, these added syllables also tended to be variable from one rendition to the next and could not be readily characterized by a small and fixed set of exemplars.

Introduction of abnormal material into the songs of deafened birds was observed in approximately half of the cases in which there was also some recognizable preservation of the original syllables. In these cases, individual bouts of song were often initiated with the most normal vocalizations, and abnormal syllables only subsequently appeared in the bout (as in the examples of Fig. 4). This gave the impression that songs became increasingly disrupted as singing progressed. The stretches of disorganized and abnormal vocalizations were often interrupted by relatively loud, call-like vocalizations, after which another comparatively normal sequence of syllables could be produced (Fig. 4c).

Another feature that characterized song changes after deafening was that the frequency control of individual syllables appeared to deteriorate; the fundamental frequency wavered abnormally rapidly, and the overall appearance of syllables was noisier. This was especially clear for some syllables that remained recognizable after deafening and therefore could be followed over time. For example, the terminal component of syllable " $\mathrm{d}$ " in Figure $4 a$ was a harmonic stack with a smoothly varying fundamental frequency before deafening. At $41 \mathrm{~d}$ after deafening this same component of the syllable exhibited rapid fluctuations in the fundamental frequency (and its harmonics) so that it had a more "wavery" appearance in the spectrogram (Fig. 4c). By the final recording from this bird, at $203 \mathrm{~d}$ after deafening, this waveriness was even more pronounced, and the harmonics were less clearly separated, so that the syllable had a noisier overall appearance (Fig. 4d). Although it was especially clear in cases in which individual syllables could be followed, this type of deterioration of frequency control was a general feature of the changes in song that followed deafening. That is, there was an apparent tendency for the overall quality of the song to become more noisy and for individual syllables to exhibit more waveriness after deafening.

As observed for several of the younger control birds, this bird's song also speeded up. The total duration of the sequence "abcd" decreased from $578 \mathrm{msec}(\mathrm{SD}=10)$ at $103 \mathrm{~d}$ of age to $535 \mathrm{msec}$ $(\mathrm{SD}=8) 41 \mathrm{~d}$ after deafening and $489 \mathrm{msec}(\mathrm{SD}=15)$ in the final recording $\sim 6$ months later. The speeding up was generally more pronounced in the deafened birds than in the controls (see below for quantification), suggesting that the underlying mechanisms for the speeding up of song not only did not require auditory feedback but were actually accentuated in the absence of feedback.

\section{Old deafened birds}

Another example of comparatively moderate changes in song that occurred after deafening of an older bird is shown in Figure 5. This bird was deafened at $395 \mathrm{~d}$ of age. Before deafening (Fig. $5 a$ ), the bird had a very stereotyped motif ("bcdefg") that was typically sung twice in immediate succession. Shortly after deafening (4 d post), the song was essentially unchanged (Fig. 5b). Similarly, at $34 \mathrm{~d}$ after deafening, the bird continued to produce all of the syllables of its initial repertoire (Fig. 5c). However, the syllable "g" was only very rarely produced, so that most motifs ended abnormally early (Fig. $5 c$, arrows; the probability of a "g" after an "f" had dropped from an initial value of $100 \%$ to a value of $25 \%$ ). Moreover, when the syllable "g" was produced, its spectral structure was noticeably degraded. Also at this time, the song no longer linked together two motifs in immediate succession; instead there were often pauses of up to several hundred milliseconds, further suggesting some difficulty in proceeding normally through the terminal portion of the motif. By $94 \mathrm{~d}$ after deafening (Fig. $5 d$ ), this apparent difficulty was even more pronounced; the bird continued to produced a truncated motif (lacking the last syllable "g" in $>95 \%$ of motifs), and on the rare 
occasions when the syllable "g" was produced, it was further degraded, exhibiting a noisy spectral structure. The truncated motif was almost always followed by a series of abnormal, lowamplitude, and variable syllables (*), before another partial motif was initiated. These stretches of abnormal vocalizations often included syllables with unusually short durations and intervals with unusually long durations, relative to less disrupted portions of song. Other abnormalities of sequence (arrows) were also apparent at this time; for example, syllables occasionally were produced in sequences that were never observed before deafening (e.g., the transition from "c" to "e"). In the final recording from this bird at $256 \mathrm{~d}$ after deafening, these types of changes in song were further accentuated (Fig. 5e). Although some of the original syllables remained essentially unchanged, some (e.g., "c" and "g") were never observed, and others (e.g., "f") were appreciably altered. There also continued to be stretches of abnormal, short-, and low-amplitude syllables (*) interposed between sequences of recognizable syllables. Furthermore, the sequences in which the remaining recognizable syllables were sung frequently included additional transitions that were never observed before deafening (e.g., "a" to "d", "b" to "d", "c" to "d", and "i" to "b", indicated by Fig. $5 e$, arrows).

\section{Summary of qualitative changes to song after deafening}

The preceding examples illustrate the range of effects on song that we observed after deafening. The changes in song were clearly quite variable across birds. In the most extreme cases, all recognizable aspects of the original song were obliterated within the first few weeks after deafening (Fig. 3). In other cases, songs retained some or all of their original syllables in recognizable form for as long as birds were followed after deafening (up to $\sim 1$ year; Figs. 4, 5). However, even the least seriously affected birds eventually developed conspicuous abnormalities in their songs. These often included a deterioration in the spectral structure of recognizable syllables such that they became more noisy (with a more uniform distribution of energy across frequency) or developed rapid fluctuations in frequency (i.e., syllable "d" in Fig. 4, and syllables "g" and "f" in Fig. 5). These abnormalities could also include changes to the temporal structure of the song, such as the introduction of variable new syllables $(*)$, as well as the introduction of atypical transitions between recognizably retained syllables.

\section{Stabilization of song by AFP lesions and age}

In the following sections of Results, we characterize more systematically the changes in song that occurred for the entire population of birds in this study, and we examine whether and how the degree of song changes varied with the age of deafening and with lesions of the anterior forebrain pathway. This characterization is divided into two components, one focusing on changes to the individual syllables of the bird's repertoire, and the other on changes to the overall temporal pattern of the song. This division was chosen both to make analysis of the data more tractable and because physiological experiments suggest that different components of the song motor control system govern these two aspects of song in zebra finches ( Vu et al., 1994; Yu and Margoliash, 1996; Margoliash, 1997).

\section{Changes to the structure of individual syllables}

To grossly assess changes to syllables after deafening, we scored the extent to which individual syllables that were initially present in each bird's repertoire were retained in subsequent recording

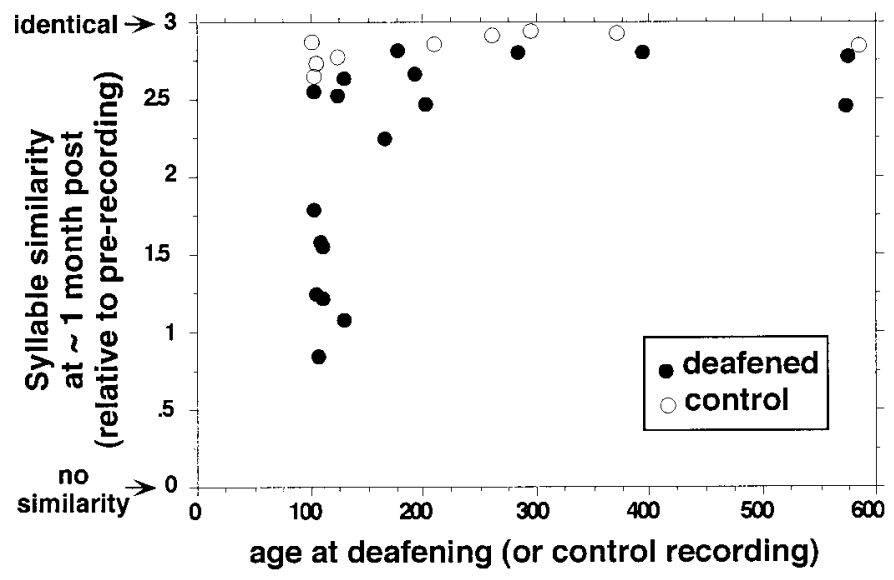

Figure 6. Changes to syllable similarity after deafening. Summary for all birds of the average similarity between syllables initially present in each bird's repertoire and syllables present 1 month after deafening (solid circles) or, for control birds (open points), 1 month after the initial recording. Data are plotted as a function of the age at which birds were deafened or, for control birds, as a function of the age at which song was initially recorded.

sessions, using a method similar to that previously used in studies of song (Nordeen and Nordeen, 1992; Morrison and Nottebohm, 1993; Lombardino and Nottebohm, 2000). Human observers who were familiar with zebra finch song, but were unaware of the conditions of the experiment, were asked to judge the similarity between syllables from songs recorded on two different dates, scoring the quality of that match on a scale of $0-3(0=$ no similarity; 3 = identical). These scores were averaged across all syllables sung by an individual bird to derive a mean "syllable similarity" value for each bird, representing the degree to which syllables that were initially present were retained (see Materials and Methods for more details).

We first assessed the entire population of intact deafened birds at $\sim 1$ month after deafening $(n=18)$. Consistent with the results of Lombardino and Nottebohm (2000), we found that the deterioration of syllable structure depended on the age at which birds were deafened. Figure 6 summarizes the degree of syllable similarity for all deafened birds as a function of the age at which deafening occurred. Also shown for comparison are data from control birds indicating the degree of syllable similarity $\sim 1$ month after an initial recording as a function of the age of the bird at initial recording. It is apparent that whereas the control birds retained a high degree of syllable similarity over time, the deafened birds exhibited a variable degree of syllable deterioration that was clearly most pronounced for the younger adults.

We then asked whether the syllable deterioration at 1 month of after deafening continued to progress with time and whether any age-dependent differences persisted over long periods after deafening. We also wanted to compare the degree of protection from deterioration provided by age with that imparted by AFP lesions. For the analyses that follow, we therefore divided the deafened birds into three groups: "young-deafened" birds, deafened between 100 and $130 \mathrm{~d}$ of age $(n=11)$, "old-deafened" birds, deafened at $>165 \mathrm{~d}$ of age $(n=8)$, and "lesioned-deafened" birds, lesioned immediately before deafening, between 100 and $130 \mathrm{~d}$ of age $(n=6)$. For these groups, we quantified song changes at two time points: an early time point ( $\sim 1$ month after deafening), and a late time point, representing the final recording from each bird (at least 6 months after deafening). Because recordings 
Figure 7. Overall summary of changes to syllable similarity of song at 1 month and final time points for each experimental group. Each point corresponds to an individual bird and indicates the average similarity (see Materials and Methods) between syllables from the repertoire present in the initial recording session and syllables present $\sim 1$ month or $>6$ months after the indicated manipulations. Bars indicate group means and SEs. Significant differences between groups are indicated in Table 1 . Control birds (cont) and deafened birds (deaf) were each divided into two groups, based on age at initial recording: young adults $(Y)$ were between 100 and $130 \mathrm{~d}$ of age, and old adults $(O)$ were between $165 \mathrm{~d}$ and 2 years of age. Data from the young-deafened birds are indicated by filled bars, and data from the old-deafened birds are indicated by striped bars. Birds in the "lesion" group (shaded bars) received bilateral electrolytic lesions of nucleus LMAN immediately before deafening. Birds in the "sham" group received lesions outside of the song system before deafening. All of the lesioned birds except for one (indicated by the square) were young adults at the time of the manipulation. Data from two other groups are shown for comparison: the random group indicates the average similarity present between the syllables of unrelated birds, and the nerve cut group indicates the average similarity between syllables sung before and a few days after denervation of the bird's vocal organ by bilateral transection of the tracheosyringeal nerve (Fig. 1). These two groups provide measures of the minimal expected degree of syllable similarity (dashed line).

were not available from all birds at both time points, the numbers for each analysis and experimental group varied as noted in Table 1. This table also summarizes the values of a variety of measures, discussed in further detail below, that were applied to the experimental groups and indicates differences between groups that were significant.

Figure 7 shows changes in syllable similarity for each experimental group. An ANOVA confirmed that there were significant effects of both age at deafening and time after deafening on the amount of syllable deterioration. At 1 month after deafening, young-deafened adults exhibited a significant reduction in syllable similarity relative to age-matched controls. In contrast, olddeafened birds had not yet deteriorated significantly relative to controls. For both groups of birds, however, there was a highly significant decrease in syllable similarity between 1 month after deafening and the final post-deafening recording. Indeed, for each intact deaf individual in the study, syllable similarity decreased over this period. These data indicate that syllables continue to deteriorate over a period of months after deafening. By the final recording, syllable similarity for young-deafened birds was no longer significantly different from the baseline value of 0.96 for randomly matched songs (dashed line). For old-deafened birds syllable similarity had also decreased significantly relative to age-matched controls but remained significantly greater than the value for young deafened birds. These data indicate that there was a larger and more rapid deterioration of syllables in youngdeafened birds than in old-deafened birds and that the difference between these groups persisted for at least 6 months after deafening.

In contrast, lesioned-deafened birds (young adult birds that received bilateral lesions of LMAN just before deafening) were strikingly different from both young and old-deafened birds. The right panel of Figure $6 b$ shows that at 1 month after deafening syllables were as well preserved in the lesioned birds as in hearing controls and were significantly better preserved than in youngdeafened birds, consistent with a previous report (Brainard and Doupe, 2000). Moreover, AFP lesions did not simply confer on these birds the same degree of stabilization normally provided by age; at the final time point ( $>6$ months after deafening), when old-deafened birds exhibited a significant decrease of syllable similarity, lesioned birds still had not deteriorated relative to hearing controls.

\section{The nature of changes to syllables}

Although the "gestalt" syllable similarity measures used here and by others (Nordeen and Nordeen, 1992; Lombardino and Nottebohm, 2000) provide a general sense of the degree to which syllable structure is altered, they do not characterize the nature of that change. In principle, different aspects of syllable structure can change independently, and an understanding of which aspects change after deafening may provide insights into which parts of the song system are affected. We therefore used several measures to assess the structure of song syllables as birds matured, the degradation of syllables after deafening, and the dependence of this degradation on age and the AFP.

One general finding was that syllables appeared gradually to become noisier after deafening; that is, the harmonic structure of syllables became less well defined, with more uniform distribution of energy across frequency. In cases in which it was possible to follow individual syllables over time, we used the depth of frequency modulation of identified harmonic components of syllables as a quantitative measure of noisiness (see Materials and Methods). The changes in noisiness as assessed by this measure are summarized for all groups of birds in Figure $8 A$. Five youngdeafened birds and one old-deafened bird were excluded from this analysis because no syllables remained unambiguously identifiable. For the control birds, noisiness did not increase over time. Indeed for the young controls there was a small but significant decrease in noisiness between the first and last recording sessions, consistent with the possibility that there is normally a subtle refinement of syllable structure in early adulthood. In contrast, deafened birds exhibited large increases in the noisiness of syllables.

We also used a subjective scoring procedure to assess the impression that syllable frequency structure became noisier after deafening. Observers who were familiar with zebra finch song but 

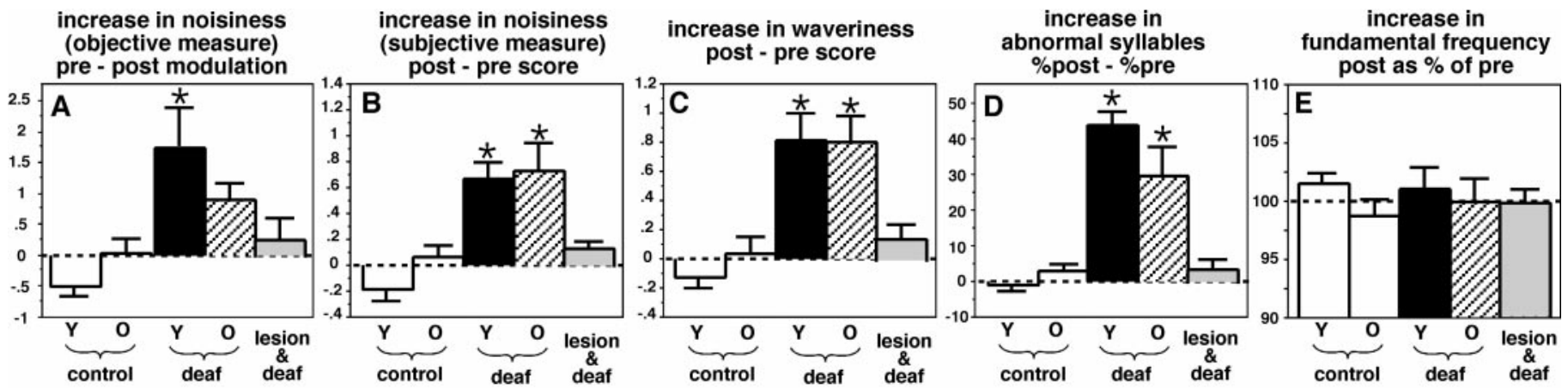

Figure 8. Nature of changes to syllables after deafening. Each bar indicates the mean change $( \pm \mathrm{SE})$ between the first and final recording session $(>6$ months later) for all birds in the indicated groups. $A$, Changes in syllable noisiness as characterized by depth of modulation of harmonic spectral structure (see Materials and Methods) for syllables that remained identifiable over time. Five young-deafened birds and one old-deafened bird were excluded because of a lack of retained syllables. $B, C$, Changes in noisiness and waveriness scores of spectral structure (see Materials and Methods). These measures were applied to a random sample of 50-100 syllables from each recording session. $D$, Changes in percentage of syllables that were judged to be abnormal based on their spectral structure. $E$, Changes in fundamental frequency. Asterisks indicate significant differences from age-matched controls and from birds that received lesions before deafening.

blind to the treatment of birds scored individual syllables for their overall degree of noisiness (see Materials and Methods). This procedure was applied to a random sampling of syllables from each bird and recording session. It therefore differed from the quantitative procedure above in that it could be applied to cases in which individual syllables were no longer recognizable and was also sensitive to the introduction of new syllables to song. Figure $8 b$ shows the average change in noise scores between the first recording session (immediately before any manipulation) and the final recording session ( $>6$ months later) for the different groups. The control birds showed no significant change in noisiness of syllables, although there was again a trend for these measures to decrease slightly in the young controls. In contrast, deafening caused a large and significant increase in this measure. These two measures thus confirm the qualitative impression from the examples shown earlier that deafening increases the noisiness of syllables.

A second change to syllables that followed deafening was an increase in the waveriness of spectral structure; that is there was a tendency to produce syllables with abnormally rapid fluctuations in frequency. Figure $8 c$ summarizes changes to the waveriness of syllables (see Materials and Methods) for each of the experimental groups. Control birds again tended to sing slightly less wavery syllables as they got older, although this improvement was not significant, whereas deafening caused a large and significant deterioration of this aspect of frequency control.

A third attribute of song that was scored by observers was whether individual syllables were abnormal in structure. Syllables that were scored as abnormal included ones with conspicuous upwardly sweeping frequency components (Fig. $3 f$, bold arrows), very short duration interruptions (Fig. 3f, open arrows), high levels of noise, and extreme waveriness. The percentage change in abnormal syllables for each experimental group is shown in Figure $8 d$. Again, as suggested by the examples shown earlier, there was a significant introduction of abnormal syllables only to the songs of the deafened birds.

Finally, one aspect of human speech that has been reported to change systematically after profound hearing loss in adulthood is pitch (Leder et al., 1987a; Cowie and Douglas-Cowie, 1992). For birdsong, pitch can be characterized by the fundamental frequency of syllables with harmonic spectral structure. A previous study found no change to the fundamental frequency of zebra finch syllables over a 16 week period after deafening, but suggested that a change in frequency might have developed had the birds been followed for longer (Nordeen and Nordeen, 1992). We therefore assessed whether any systematic changes to the fundamental frequency of syllables occurred between the initial and final recordings ( $>6$ months later). We measured the fundamental frequency of identified syllables (one to five per bird) that had well defined harmonic structure and that could be followed over time. Figure $8 e$ shows the average percentage change in fundamental frequency for the different groups of birds. Despite the overall deterioration of spectral structure after deafening, we found no net change in fundamental frequency.

For each of the above measures of syllable structure, we also assessed the magnitude of changes in young adult birds that received lesions of LMAN before deafening. The lesioned birds were significantly less affected by deafening than were the young or the old intact deafened birds. Indeed, there was no significant difference between the lesioned birds and their age-matched controls. These data indicate that lesions of LMAN significantly prevent the deterioration of spectral structure and introduction of abnormal syllables that normally follow deafening in both young and old adults.

Although birds that received AFP lesions before deafening did not differ significantly from age-matched controls for any measure of change in syllables, there was nevertheless a (nonsignificant) tendency for measures of syllable structure to change more than in controls. This tendency might simply reflect the contribution of "lesioned" birds that received incomplete lesions of LMAN. There was not, however, a significant correlation between the sizes of lesions and the magnitude of changes to song. Alternatively, some of the tendency for subtle changes to syllables in the lesioned group might reflect changes in the periphery of the motor pathway, such as strengthening or atrophy of vocal muscles, that can neither be blocked by AFP lesions nor corrected without auditory feedback.

\section{Changes to the temporal structure of song}

In addition to the acoustic structure of syllables, song, like speech, has numerous global temporal features, including the sequence and temporal pattern of syllables, the duration of individual syllables and intervals, and the overall rate of delivery of the song ("tempo"). These aspects of song may be controlled by brain 
Figure 9. Summary of changes to the temporal pattern of song. Each point corresponds to an individual bird and indicates the similarity between the temporal pattern (see Materials and Methods) of songs from the initial recording session and songs recorded $\sim 1$ month or $>6$ months after the indicated manipulations. Conventions are as in Figure 7.

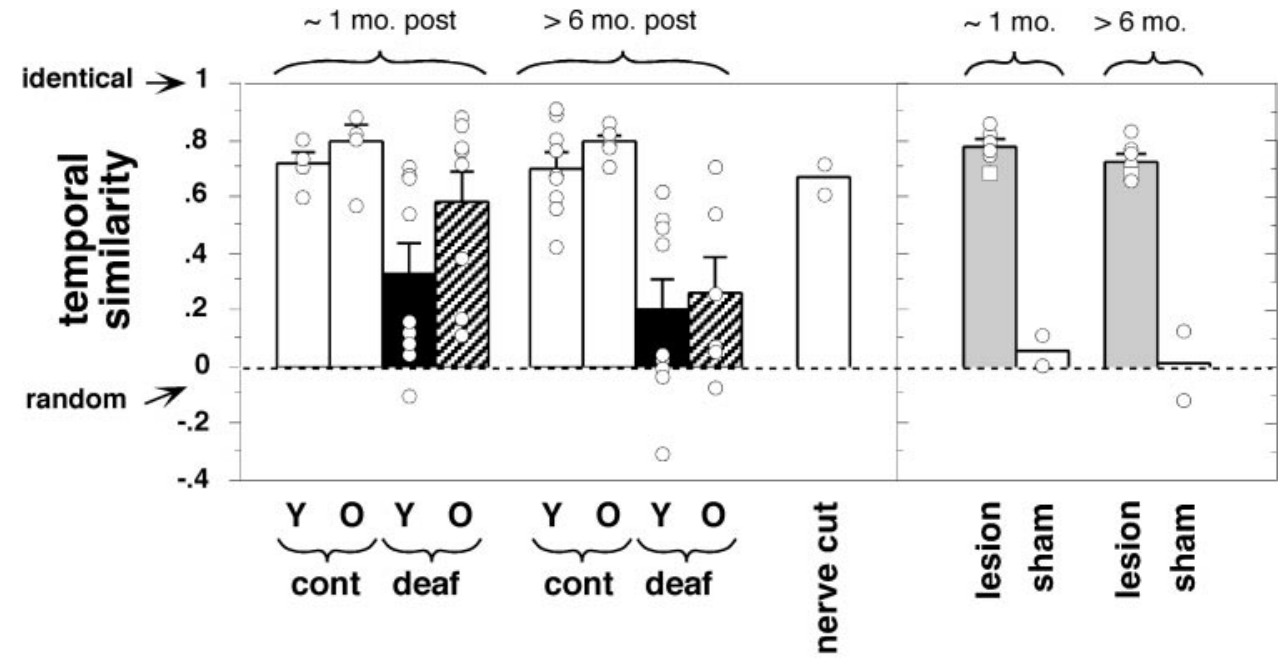

regions different from those controlling syllable structure. Indeed, the effects of AFP lesions on deafening-induced changes in temporal structure did not exactly parallel the effects of these lesions on changes in syllables.

\section{Changes to temporal patterning}

To assess changes to the patterned temporal structure of the song of all birds, even when syllables were too deteriorated after deafening to be recognized for sequence analysis, we used a quantitative measure based on cross-correlation analysis (see Materials and Methods). This measure assessed the degree to which the initial timing of syllables within the bird's motif was preserved in later songs and explicitly ignored any changes to the spectral structure of individual syllables. Moreover, because some songs speeded up over time (see below), this measure allowed for "proportional" changes in the temporal pattern of motifs (i.e., without change in the relative durations of syllables and intervals). This measure showed that the temporal structure of song deteriorated after deafening in a manner similar to that for syllable structure (Fig. 9). At 1 month after deafening, changes were highly variable among intact deaf individuals; some birds exhibited no more change in temporal pattern than was present for controls, whereas others already exhibited a maximal degree of deterioration. However, as a group, young-deafened birds exhibited significant deterioration of temporal pattern relative to controls, whereas old-deafened birds did not. By the final recording session, the temporal pattern for both young- and old-deafened groups had further deteriorated and no longer preserved greater temporal similarity to predeafening songs than to random songs. In contrast, birds that had been lesioned as well as deafened were still not significantly different from controls, indicating that LMAN lesions stabilized the temporal pattern of song to a greater extent than did age.

In principle, changes to the temporal pattern of song can occur independently of deterioration of syllable structure. This occurs, for example, after transection of the tracheosyringeal nerve, which is essential for controlling the structure of individual syllables but not for the timing of expiration during song. Such nerve transection in two birds caused an acute loss of syllable similarity without grossly affecting the temporal pattern of song (Figs. 7, 9; "nerve cut"). Despite this dissociability of song changes, there was nevertheless a correlation between the degree of deterioration of syllables (as assessed by the syllable similarity measure) and the degree of deterioration of temporal pattern in the songs of birds after deafening $\left(R^{2}=0.4 ; p<0.05\right.$ that the slope of the regression is 0 ).

These results indicate that, like the structure of individual syllables, the temporal pattern of song deteriorates in an agedependent manner after deafening. Birds deafened as young adults exhibited larger and more rapid deterioration of temporal pattern than did birds deafened at greater ages. However, deaf birds of all ages eventually exhibited significant deterioration of temporal pattern relative to controls, and this deterioration was prevented in birds that received lesions of the AFP.

\section{Changes to average syllable and interval durations}

The data above demonstrate that the temporal pattern of the bird's original motif can be disrupted after deafening. However, this measure explicitly ignores changes to the tempo of song elements (see Materials and Methods) and also does not characterize changes to the temporal structure of song that occur outside the context of the motif, such as the introduction of short duration syllables to songs in which the original motif is well preserved (Fig. 4) or the lengthening of pauses that occur between motifs or within stretches of abnormal vocalizations (Figs. $3-5)$. As a gross measure of the nature of overall changes to the temporal structure of song, we therefore also measured changes in the average durations of syllables and intervals. This was a population measure of "unmatched" song elements in which all incidences of all syllables (or intervals) were included (see Materials and Methods). Figure 10 shows the percentage changes in mean syllable and interval durations between the first and final recording sessions for each group of birds. For the control birds, there was little change in the average duration of syllables or intervals, although there was a tendency for these elements to shorten in young control birds. In contrast, for both young- and old-deafened birds there was a large decrease in syllable durations and a large increase in interval durations, and these changes were significantly greater than those observed for age-matched controls. The direction and magnitude of these deafeninginduced changes were qualitatively in agreement with a previous study that used the same measure to characterize changes to the songs of adult birds after deafening (Wang et al., 1999). Lesions of LMAN did not prevent decreases in average syllable duration. However, they did significantly prevent increases to the average duration of intervals. The different direction of deafening- 
A unmatched syllables

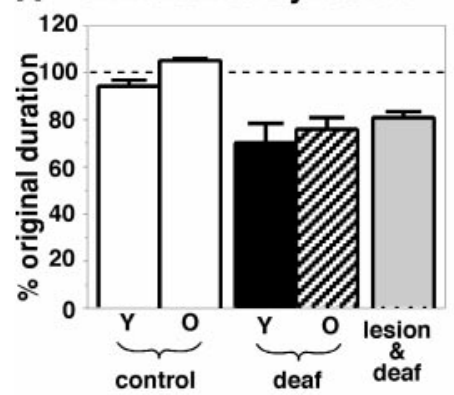

B unmatched intervals

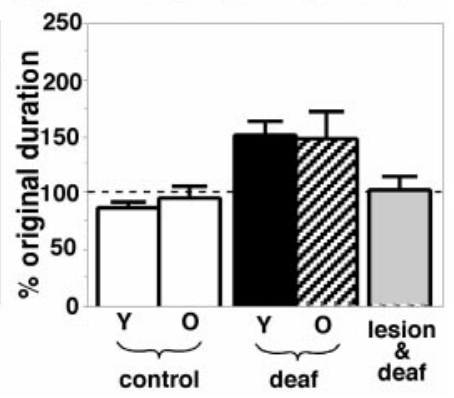

Figure 10. Average change in duration between the first and the final recording session for unmatched syllables $(A)$ and intervals $(B)$. For each bird and recording session the average duration of all syllables from at least 10 songs was calculated as well as the average duration of all intervals $<500 \mathrm{msec}$ in duration. The average duration from the final recording session was expressed as a percentage of the average duration from the initial recording session for each bird. Bars indicate the mean $( \pm \mathrm{SE})$ change in durations for all birds in each group.

induced changes in the duration of syllables versus intervals and the different effects of LMAN lesions on these changes suggest that separate factors underlie these changes.

\section{Changes to song tempo}

A variety of different factors may have contributed to changes to mean unmatched syllable and interval durations. For example, mean syllable durations could decrease either if individual syllables were sped up (Fig. 2, control bird; Fig. 4, deafened bird) or if more short duration syllables were sung relative to the number of long duration syllables (Fig. 5, deafened bird). To determine whether changes to song tempo contributed to changes in the average durations of all (unmatched) syllables and intervals, we measured changes in the duration of matched sequences of syllables that were retained between initial recordings and recordings made at $\sim 1$ month and $>6$ months after experimental manipulations (Fig. 11). Five young-deafened birds and one old-deafened bird were excluded from this analysis because original sequences of syllables were not sufficiently preserved. The young control birds exhibited a small but significant increase in song tempo. By 1 month after the initial recording, the durations of matched sequences were shortened on average by $1.5 \%$ of their initial values, and by the final recording session the durations had shortened by $7 \%$ of their initial values. This finding is consistent with the observation of Arnold (1975) that songs of normal zebra finches can speed up after $90 \mathrm{~d}$ of age and indicates that there continue to be subtle changes to normal song beyond the nominal age of song "crystallization."

The speeding up of song was accentuated in young-deafened birds. By 1 month after deafening the average duration of identified sequences had shortened by $6 \%$, and by the final recording session it had shortened by $18 \%$. This increase in tempo was significantly greater than that in young control birds, indicating that removal of auditory feedback contributed to the acceleration of song. A couple of the old-deafened birds also exhibited large increases in tempo. However, on average, deafening did not significantly speed up the songs of old-deafened birds relative to age-matched controls. This indicates that aging decreases the effects on song tempo of removing auditory feedback. In contrast, lesions of the AFP did not prevent increases in song tempo; lesioned-deafened birds exhibited increases in tempo that were as great as those that occurred in intact young-deafened birds

\section{matched sequences}

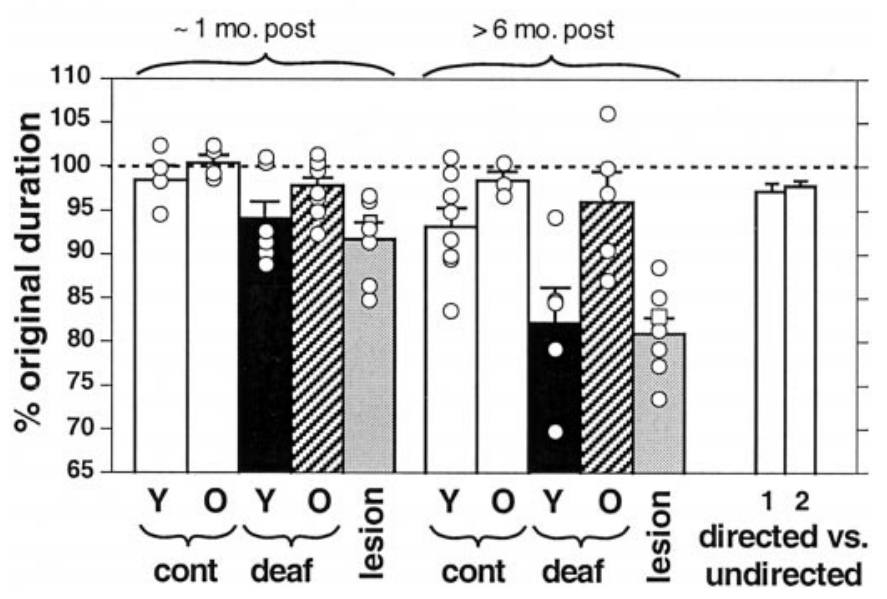

Figure 11. Change in duration of matched sequences of syllables. Points correspond to individual birds and indicate the durations of identified sequences of syllables at the 1 month and final time points as a percentage of the durations of those same sequences at the initial recording. Bars indicate mean $( \pm \mathrm{SE})$ values for all birds from each group. For comparison, data are shown illustrating the average decrease in duration of identified sequences for songs sung to another bird (directed) versus songs sung in isolation (undirected). These data derive from (1) eight birds in a published study (Sossinka and Bohner, 1980), and (2) four old control birds from the current study that were recorded in both social contexts.

and that were significantly greater than those that occurred for control groups or for old-deafened birds.

Changes to the tempo of matched sequences could reflect changes to the durations of the syllables that make up those sequences, the intervals within the sequences, or both. We therefore separately analyzed percentage changes to these two components of the total duration of the sequences used to assess tempo (matched syllables and matched intervals). For control birds, there was a significant correlation between changes to matched syllable durations and corresponding sequence durations (Fig. $12 a ; R^{2}=0.77$ ) as well as between changes to matched interval durations and sequence durations (Fig. 12b; $R^{2}=0.52$ ). These data indicate that changes to both syllables and intervals contributed to the overall acceleration of song tempo in control birds. For deafened birds, there was also a significant correlation between changes to syllable durations and sequence durations (Fig. $12 c ; R^{2}=0.86$ ). However, there was not a correlation between changes to interval durations and sequence durations for these birds (Fig. $12 d ; R^{2}=0.005$ ). This indicates that the increases in tempo that occurred after deafening were primarily because of a shortening of syllables.

Small tempo changes normally occur in the songs of zebra finches in response to alteration of social context (Sossinka and Bohner, 1980; Hessler and Doupe, 1999a); song is generally faster when sung to other birds in a social setting ("directed" song) than when sung in isolation ("undirected" song). We therefore compared the magnitude of tempo changes described here to tempo changes that occur in response to changes in social context. For four control birds from our study we found that the tempo of directed song was on average $2.1 \%$ faster than for undirected song, similar to the $2.7 \%$ change reported by Sossinka and Bohner (1980; Fig. 11, directed vs undirected). The tempo changes for young control, young-deafened, and lesioned-deafened birds were therefore much larger than the tempo changes induced by altering social context. They also differed from socially induced 

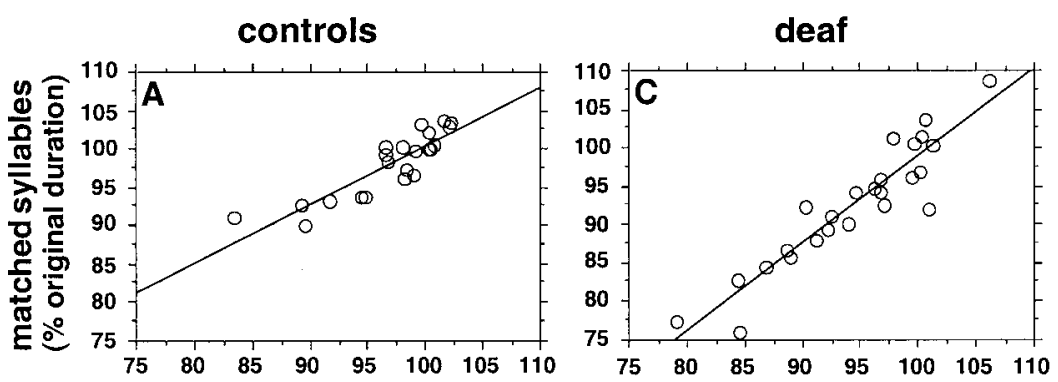

Figure 12. Contributions of syllable and interval changes to overall changes in song tempo. $A$, Correlation for control birds between the change in total duration of entire identified sequences that were preserved over time and the change in duration of the matched syllables from those sequences. $B$, Correlation for control birds between the change in total duration of entire identified sequences that were preserved over time and the duration of the matched intervals from those sequences. $C$, Correlation for deafened birds between changes to total duration and changes to syllable duration. $D$, Correlation for deafened birds between changes to total duration and changes to interval duration.
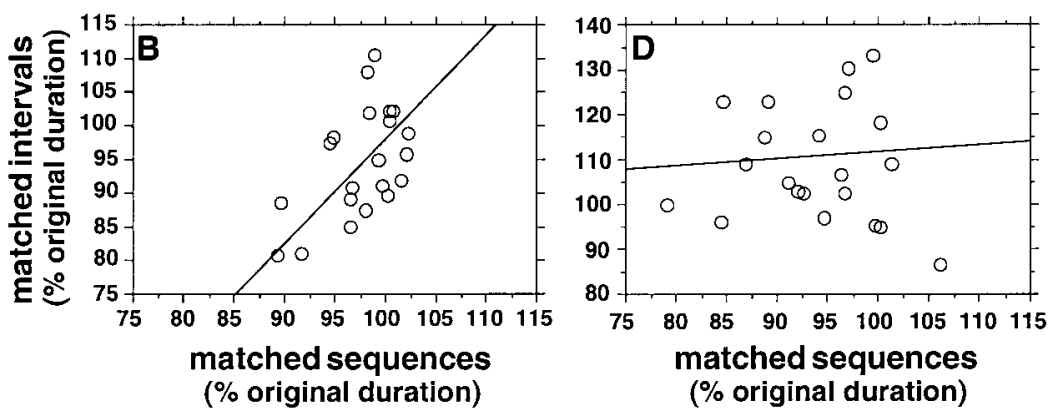

tempo changes in that they developed much more gradually (weeks vs seconds). Thus, the mechanisms that underlie acceleration of song tempo in our experimentally manipulated birds are unlikely to be the same as those that underlie social modulation of song tempo.

These data, based on a consideration of matched sequences of syllables, indicate that fixed portions of the song motor program can be accelerated over time in deafened and lesioned birds. This acceleration does not, however, fully account for the observed changes to all (unmatched) syllable and interval durations. Figure $13 a$ illustrates that, for the intact deafened birds, there were much greater reductions in syllable durations when all syllables, rather than just matched syllables, were considered. This is consistent with the observation that when syllables are present in postdeafening songs that cannot be directly matched to syllables from the predeafening repertoire, those syllables tend to be of short duration (e.g., most postdeafening syllables in Fig. 3 and syllables marked with an asterisk in Figs. 4 and 5). Likewise, Figure $13 b$ illustrates that there was a much larger increase in interval durations for the deafened birds when all intervals, rather than just matched intervals, were considered. This is consistent with the observation that intervals present in postdeafening songs that occurred outside the context of identified sequences, for example between motifs or between new abnormal syllables, tended to be of long duration (Figs. 3, 4).

\section{DISCUSSION}

\section{The nature of deafening-induced changes to song}

Our analyses of the effects of deafening on adult zebra finch song generally confirm and extend previous studies (Price, 1979; Nordeen and Nordeen, 1992; Wang et al., 1999; Brainard and Doupe, 2000; Lombardino and Nottebohm, 2000). In addition to showing gross changes to syllables as assessed by gestalt measures (Figs. 6, 7; Nordeen and Nordeen, 1992; Lombardino and Nottebohm, 2000), we demonstrate specific changes to spectral structure that consistently occur after deafening, including increased noisiness and reduced control of fundamental frequency (Fig. 8). We also show that, in addition to changes to durations of individual song elements (Fig. 10; Nordeen and Nordeen, 1992; Wang et al., 1999;
Lombardino and Nottebohm, 2000), there are changes to global temporal properties of song, including a deterioration of the temporal pattern in which syllables are produced (Fig. 9), and, for young-deafened birds, a systematic acceleration of song tempo (Figs. 11, 12).

These acoustic changes to song after deafening have several parallels to deterioration of human speech after profound hearing loss in adulthood. For example, spectral properties of speech can be degraded such that fundamental frequency varies abnormally over time and spectra of individual syllables become flattened and noisy (Kirk and Edgerton, 1983; Lane and Webster, 1991; Cowie and Douglas-Cowie, 1992; Lane et al., 1997). Likewise, sequential organization of speech can be disrupted such that syllables are inappropriately dropped, repeated, or substituted one for another. Moreover, the temporal pattern of speech may be further altered by speeding up or slowing down, by an increase in the duration of pauses between syllables and by inappropriate voicing during normally silent periods (Penn, 1955; Kirk and Edgerton, 1983; Leder et al., 1987b; Lane and Webster, 1991; Cowie and Douglas-Cowie, 1992).

\section{Stabilization of song by age and by AFP lesions}

Both age and lesions of the AFP nucleus LMAN stabilize adult song (Brainard and Doupe, 2000; Lombardino and Nottebohm, 2000). For most measures in this study, we found that AFP lesions stabilize song to a significantly greater extent than does age; whereas old-deafened birds eventually exhibited significant deterioration of song, lesioned-deafened birds generally did not differ from controls. The one exception (discussed below) was for song tempo, which was significantly stabilized by age, but not by AFP lesions. The results indicate that LMAN lesions do not simply mimic a normal aging process. Moreover, the greater stability of song of AFP-lesioned birds versus song of old-deafened birds suggests that the AFP continues to play a role even in the residual song plasticity that is observed after age-dependent stabilization of song.

The age-dependent stabilization of song implies that after the phenomenological attainment of adult song, there nevertheless continue to be changes within the nervous system that render 
A
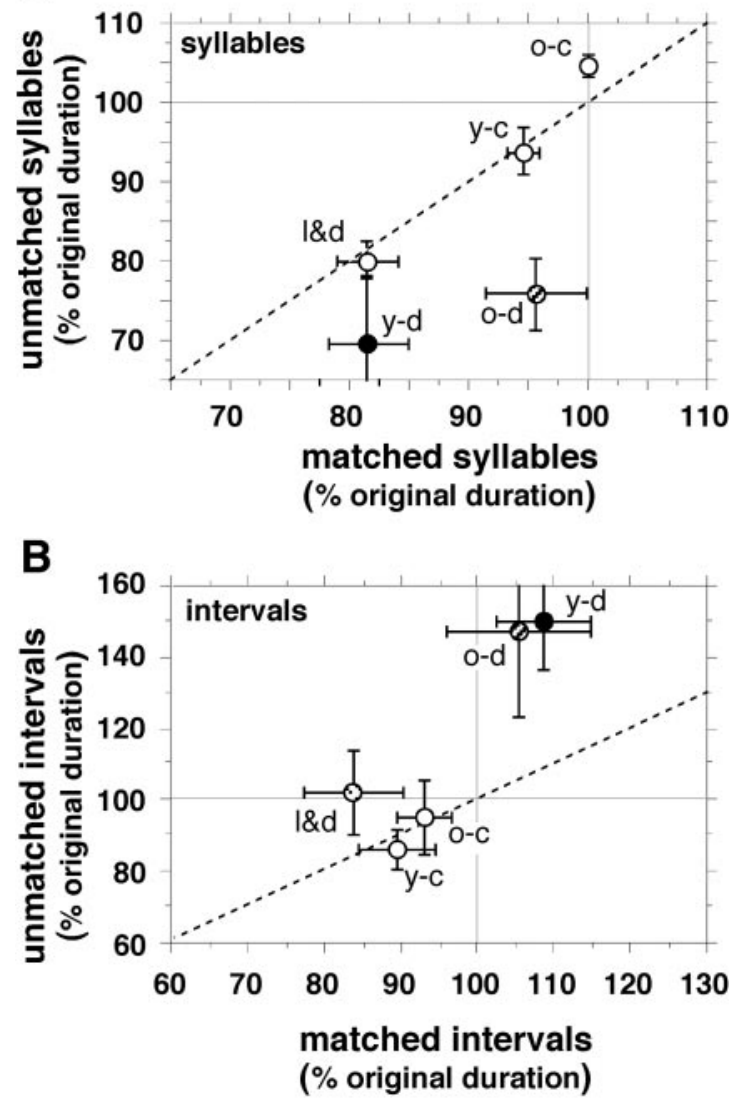

Figure 13. Comparison of changes to durations as assessed by consideration of all song elements, regardless of their identity (unmatched), or only song elements that were recognizably retained (matched). For syllables $(A)$, both measures yielded similar results for the young control $(y-c)$, old control $(o-c)$, and lesioned and deafened $(l \& d)$ groups. However, for the young-deafened $(y-d)$ and old-deafened $(o-d)$ groups, there was a greater decrease in duration when unmatched syllables were considered than when only matched syllables were considered. For intervals $(B)$, both measures again yielded similar results for the control and lesioned groups, but for the deafened groups, increases in interval durations were greater when unmatched intervals were considered than when only matched intervals were considered.

song progressively less sensitive to the removal of auditory feedback. This kind of postlearning decline in susceptibility of behavior to disruption occurs in other cases of perceptual and motor learning and is often described as a process of memory consolidation (Dudai, 1996). For example, learned arm movements become progressively less disrupted by a conflicting task over a period of several hours after achievement of asymptotic performance (Brashers-Krug et al., 1996; Shadmehr and Brashers-Krug, 1997). Consolidation over a longer time period, such as occurs for zebra finch song, also shares similarity with "sensitive" or "critical" period learning in other systems (Bolhuis, 1991; Goodman and Shatz, 1993; Brainard and Knudsen, 1998; Doupe and Kuhl, 1999). A particularly striking parallel to birdsong is provided by human speech. After initial language acquisition, speech continues to be subject to deterioration after hearing loss but becomes progressively less susceptible to disruption over a period extending for many years (Cowie and Douglas-Cowie, 1992). Thus, birdsong and speech both appear to be subject to a period of consolidation extending well beyond the age of initial learning.

An important question is whether song consolidation depends on developmental events that coincide with the completion of song learning or on the process of learning itself. Lombardino and Nottebohm (2000) suggest that song may become progressively "engrained" by each repetition of the learned motor program. Although our results do not rule out this possibility, the effects of AFP lesions indicate that the practice of learned song per se is not required for the stabilization of song. That is, song can be prematurely stabilized by lesions in a manner that is similar in many respects to the age-dependent stabilization of song, without the necessity of repeatedly singing.

\section{Possible mechanisms underlying song plasticity and consolidation}

At least two types of processes, not mutually exclusive, could contribute to the deterioration of song after deafening. (1) Feedback may normally be required to correct for ongoing perturbation of the motor program. In this case, deafening may allow effects of such perturbation to accumulate, leading to a gradual "drift" of the motor program. One possible source of such perturbation is the birth and death of neurons in song motor nuclei (Nordeen and Nordeen, 1988; Wang et al., 1999; Scott et al., 2000). (2) Deafening may actively induce perturbation of the motor program. This could occur via indirect effects of deafening on social status, hormone levels, or amounts of singing, which in turn may have physiological consequences that enhance perturbation of the motor program (Wang et al., 1999; Li et al., 2000; Livingston et al., 2000). Deafening-induced perturbation could also arise more directly from evaluation of auditory feedback; mechanisms that evaluate feedback may interpret the absence of feedback as an error of motor production and consequently generate signals that actively instruct change in the motor pathway.

The age-dependent stabilization of song may reflect some degree of "hard-wiring" of connections within the motor pathway such that they become more resistant to the types of perturbation discussed above. Additionally, this stabilization may reflect a decrease in the sources of perturbation themselves. For example, if song deteriorates because of the unguided incorporation of new neurons, then, as suggested by Lombardino and Nottebohm (2000), there may be an age-dependent decline in the birth or functional incorporation of such neurons. Similarly, if song deteriorates because of active instruction from a feedback-based error signal, then the strength or efficacy of such a signal may decline with age. This would occur if, as suggested for other systems, direct evaluation of feedback was progressively supplanted during learning by evaluation based on an internal model of performance (Jordan, 1995; Kawato, 1999; Troyer and Doupe, 2000a,b).

The stabilization of song by LMAN lesions suggests possible (not mutually exclusive) functions of the AFP. (1) The AFP may be "permissive" for motor pathway plasticity; that is, AFP integrity may be required for sources of perturbation (because of drift or because of active instruction arising from outside the AFP) to effectively alter the motor pathway. For example, if the motor pathway changes because of unguided neuronal turnover, then LMAN lesions must specifically block the birth or functional incorporation of these neurons. (2) The AFP may itself be an active source of motor pathway perturbation, providing disruptive neural or trophic signals in response to elimination of feedback. In this case, lesions of LMAN would stabilize song by removing these signals. The possibility that such signals are generated in response to a mismatch between actual feedback and desired feedback is consistent with recent studies that suggest a 
role for mammalian corticobasal ganglia circuits in feedback evaluation and error correction (Hikosaka et al., 1998; Smith et al., 2000).

\section{Changes to song tempo}

The one conspicuous change to the songs of young control birds was a gradual acceleration of song tempo. Such speeding up of production has been observed in other systems at the completion of motor learning (Hikosaka et al., 1998; Thoroughman and Shadmehr, 1999). A striking parallel from humans is a gradual acceleration of speech production that occurs over a period extending to as late as 15 years of age (Lee, 1950; Hillenbrand et al., 1995).

There was a greatly accentuated acceleration of song tempo in young-deafened birds and in birds that received lesions of LMAN before deafening (Figs. 11, 12), and similar increases in tempo occur after lesions of LMAN alone (Williams and Mehta, 1999). The data from LMAN-lesioned birds suggest that self reinforcement mechanisms operate within the motor pathway that can progressively strengthen (and accelerate) dominant patterns of singing-related motor activity, but that these mechanisms are normally held in check by signals from the AFP. Such entrainment could arise by Hebbian mechanisms if the AFP provided input to the motor pathway that was correlated with, but delayed relative to, singing-related neural activity intrinsic to the motor pathway. Indirect evidence suggests that this is the case (McCasland, 1987; Yu and Margoliash, 1996; Hessler and Doupe, 1999 b). This model could also account for the acceleration of song in deafened birds, provided that deafening degrades the correlation between activity in the AFP and song.

Although it remains unclear what mechanisms govern changes to song tempo, the involvement of the AFP is consistent with findings in other systems that basal ganglia circuits contain signals appropriate for pacing of movements and that damage to those circuits can lead to both hyperkinesias and hypokinesias of learned movements including speech (Ivry, 1996; Meck, 1996; O’Boyle et al., 1996; Ackermann et al., 1997; Gibbon et al., 1997; Plenz and Kitai, 1999).

\section{REFERENCES}

Ackermann H, Konczak J, Hertrich I (1997) The temporal control of repetitive articulatory movements in Parkinson's disease. Brain Lang 56:312-319.

Arnold AP (1975) The effects of castration on song development in zebra finches (Poephila guttata). J Exp Zool 191:261-278.

Basham ME, Nordeen EJ, Nordeen KW (1996) Blockade of NMDA receptors in the anterior forebrain impairs sensory acquisition in the zebra finch. Neurobiol Learn Mem 66:295-304.

Bolhuis JJ (1991) Mechanisms of avian imprinting: a review. Biol Rev Camb Philos Soc 66:303-345.

Bottjer SW, Hewer SJ (1992) Castration and antisteroid treatment impair vocal learning in male zebra finches. J Neurobiol 23:337-353.

Bottjer SW, Johnson F (1997) Circuits, hormones, and learning: vocal behavior in songbirds. J Neurobiol 33:602-618.

Bottjer SW, Miesner EA, Arnold AP (1984) Forebrain lesions disrupt development but not maintenance of song in passerine birds. Science 224:901-903.

Bottjer SW, Roselinsky H, Tran NB (1997) Sex differences in neuropeptide staining of song-control nuclei in zebra finch brains. Brain Behav Evol 50:284-303.

Brainard MS, Doupe AJ (2000) Interruption of a basal ganglia-forebrain circuit prevents plasticity of learned vocalizations. Nature 404:762-766.

Brainard MS, Knudsen EI (1998) Sensitive periods for visual calibration of the auditory space map in the barn owl optic tectum. J Neurosci 18:3929-3942.

Brashers-Krug T, Shadmehr R, Bizzi E (1996) Consolidation in human motor memory. Nature 382:252-255.

Cowie R, Douglas-Cowie E (1992) Postlingually acquired deafness: speech deterioration and the wider consequences. In: Trends in linguistics (Winter W, ed) Berlin: Mouton de Gruyter.
Doupe AJ, Kuhl PK (1999) Birdsong and human speech: common themes and mechanisms. Annu Rev Neurosci 22:567-631.

Dudai Y (1996) Consolidation: fragility on the road to the engram. Neuron 17:367-370.

Gibbon J, Malapani C, Dale CL, Gallistel C (1997) Toward a neurobiology of temporal cognition: advances and challenges. Curr Opin Neurobiol 7:170-184.

Goodman CS, Shatz CJ (1993) Developmental mechanisms that generate precise patterns of neuronal connectivity. Cell 72 [Suppl]:77-98.

Hessler NA, Doupe AJ (1999a) Singing-related neural activity in a dorsal forebrain-basal ganglia circuit of adult zebra finches. J Neurosci 19:10461-10481.

Hessler NA, Doupe AJ (1999b) Social context modulates singing-related neural activity in the songbird forebrain. Nat Neurosci 2:209-211.

Hikosaka O, Miyashita K, Miyachi S, Sakai K, Lu X (1998) Differential roles of the frontal cortex, basal ganglia, and cerebellum in visuomotor sequence learning. Neurobiol Learn Mem 70:137-149.

Hillenbrand J, Getty LA, Clark MJ, Wheeler K (1995) Acoustic characteristics of American English vowels. J Acoust Soc Am 97:3099-3111.

Immelmann K (1969) Song development in the zebra finch and other estrildid finches. In: Bird vocalizations (Hinde RA, ed), pp 61-74. London: Cambridge UP.

Ivry RB (1996) The representation of temporal information in perception and motor control. Curr Opin Neurobiol 6:851-857.

Jordan MI (1995) Computational motor control. In: The cognitive neurosciences (Gazzaniga $\mathrm{M}$, ed), pp 567-610. Cambridge, MA: MIT.

Kawato M (1999) Internal models for motor control and trajectory planning. Curr Opin Neurobiol 9:718-727.

Kirk MA, Edgerton BJ (1983) The effects of cochlear implant use on voice parameters. Otol Clinics N Am 16:281-292.

Konishi M (1965) The role of auditory feedback in the control of vocalization in the white-crowned sparrow. Z Tierpsychol 22:770-783.

Konishi M (1985) Birdsong: from behavior to neuron. Annu Rev Neurosci 8:125-170.

Lane H, Webster JW (1991) Speech deterioration in postlingually deafened adults. J Acoust Soc Am 89:859-866.

Lane H, Wozniak J, Matthies M, Svirsky M, Perkell J, O'Connell M, Manzella J (1997) Changes in sound pressure and fundamental frequency contours following changes in hearing status. J Acoust Soc Am 101:2244-2252.

Leder SB, Spitzer JB, Kirchner JC (1987a) Speaking fundamental frequency of postlingually profoundly deaf adult men. Ann Otol Rhinol Laryngol 96:322-324.

Leder SB, Spitzer JB, Kirchner JC, Flevaris-Phillips C, Milner P, Richardson F (1987b) Speaking rate of adventitiously deaf male cochlear implant candidates. J Acoust Soc Am 82:843-846.

Lee BS (1950) Effects of delayed speech feedback. J Acoust Soc Am 22:824-826.

Li X-C, Jarvis ED, Alvarez-Borda B, Lim DA, Nottebohm F (2000) A relationship between behavior, neurotrophin expression, and new neuron survival. Proc Natl Acad Sci USA 97:8584-8589.

Livingston FS, White SA, Mooney R (2000) Slow NMDA-EPSCs at synapses critical for song development are not required for song learning in zebra finches. Nat Neurosci 3:482-488.

Lombardino AJ, Nottebohm F (2000) Age at deafening affects the stability of learned song in adult male zebra finches. J Neurosci 20:5054-5064.

Luo M, Perkel DJ (1999) A GABAergic, strongly inhibitory projection to a thalamic nucleus in the zebra finch song system. J Neurosci 19:6700-6711.

Margoliash D (1997) Functional organization of forebrain pathways for song production and perception. J Neurobiol 33:671-693.

Marler P (1970) A comparative approach to vocal learning: song development in white-crowned sparrows. J Comp Physiol Psychol 71:1-25.

McCasland JS (1987) Neuronal control of bird song production. J Neurosci 7:23-39.

Meck WH (1996) Neuropharmacology of timing and time perception. Brain Res Cogn Brain Res 3:227-242.

Morrison RG, Nottebohm F (1993) Role of a telencephalic nucleus in the delayed song learning of socially isolated zebra finches. J Neurobiol 24:1045-1064.

Nordeen KW, Nordeen EJ (1988) Projection neurons within a vocal motor pathway are born during song learning in zebra finches. Nature 334:149-151.

Nordeen KW, Nordeen EJ (1992) Auditory feedback is necessary for the maintenance of stereotyped song in adult zebra finches. Behav Neural Biol 57:58-66.

Nordeen KW, Nordeen EJ (1993) Long-term maintenance of song in adult zebra finches is not affected by lesions of a forebrain region involved in song learning. Behav Neural Biol 59:79-82.

Nottebohm F, Stokes TM, Leonard CM (1976) Central control of song in the canary, Serinus canarius. J Comp Neurol 165:457-486. 
O'Boyle DJ, Freeman JS, Cody FW (1996) The accuracy and precision of timing of self-paced, repetitive movements in subjects with Parkinson's disease. Brain 119:51-70.

Penn JP (1955) Voice and speech patterns of the hard of hearing. Acta Otolaryngol S124:1-69.

Plenz D, Kitai ST (1999) A basal ganglia pacemaker formed by the subthalamic nucleus and external globus pallidus. Nature 400:677-682.

Price PH (1979) Developmental determinants of structure in zebra finch song. J Comp Physiol Psychol 93:268-277.

Reiner A, Medina L, Veenman CL (1998) Structural and functional evolution of the basal ganglia in vertebrates. Brain Res Brain Res Rev 28:235-285.

Scharff C, Nottebohm F (1991) A comparative study of the behavioral deficits following lesions of various parts of the zebra finch song system: implications for vocal learning. J Neurosci 11:2896-2913.

Scott LL, Nordeen EJ, Nordeen KW (2000) The relationship between rates of $\mathrm{HVc}$ neuron addition and vocal plasticity in adult songbirds. J Neurobiol 43:79-88.

Shadmehr R, Brashers-Krug T (1997) Functional stages in the formation of human long-term motor memory. J Neurosci 17:409-419.

Smith MA, Brandt J, Shadmehr R (2000) Motor disorder in Huntington's disease begins as a dysfunction in error feedback control. Nature 403:544-549.

Sohrabji F, Nordeen EJ, Nordeen KW (1990) Selective impairment of song learning following lesions of a forebrain nucleus in the juvenile zebra finch. Behav Neural Biol 53:51-63.
Sossinka R, Bohner J (1980) Song types in the zebra finch poephila guttata castanotis. Z Tierpsychol 53:123-132.

Thoroughman KA, Shadmehr R (1999) Electromyographic correlates of learning an internal model of reaching movements. J Neurosci 19:8573-8588.

Troyer T, Doupe AJ (2000a) An associational model of birdsong sensorimotor learning. I. Efference copy and the learning of song syllables. J Neurophysiol 84:1204-1223.

Troyer T, Doupe AJ (2000b) An associational model of birdsong sensorimotor learning. II. Temporal hierarchies and the learning of song sequence. J Neurophysiol 84:1224-1239.

Vu ET, Mazurek ME, Kuo YC (1994) Identification of a forebrain motor programming network for the learned song of zebra finches. J Neurosci 14:6924-6934.

Waldstein RS (1990) Effects of postlingual deafness on speech production: implications for the role of auditory feedback. J Acoust Soc Am 88:2099-2144.

Wang N, Aviram R, Kirn JR (1999) Deafening alters neuron turnover within the telencephalic motor pathway for song control in adult zebra finches. J Neurosci 19:10554-10561.

Williams H, Mehta N (1999) Changes in adult zebra finch song require a forebrain nucleus that is not necessary for song production. $\mathrm{J}$ Neurobiol 39:14-28.

Yu AC, Margoliash D (1996) Temporal hierarchical control of singing in birds. Science 273:1871-1875. 